\title{
Demystifying Black-box Learning Models of Rumor Detection from Social Media Posts
}

\author{
by \\ Faiza Tafannum \\ 17101063 \\ Mir Nafis Sharear Shopnil \\ 17101423 \\ Anika Salsabil \\ 17101498 \\ Navid Ahmed \\ 17101373
}

A thesis submitted to the Department of Computer Science and Engineering in partial fulfillment of the requirements for the degree of B.Sc. in Computer Science

Department of Computer Science and Engineering

Brac University

September 2021

(C) 2021. Brac University

All rights reserved. 


\section{Declaration}

It is hereby declared that

1. The thesis submitted is my/our own original work while completing degree at Brac University.

2. The thesis does not contain material previously published or written by a third party, except where this is appropriately cited through full and accurate referencing.

3. The thesis does not contain material which has been accepted, or submitted, for any other degree or diploma at a university or other institution.

4. We have acknowledged all main sources of help.

\section{Student's Full Name \& Signature:}

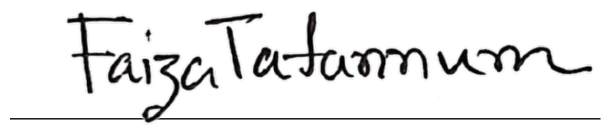

Faiza Tafannum 17101063

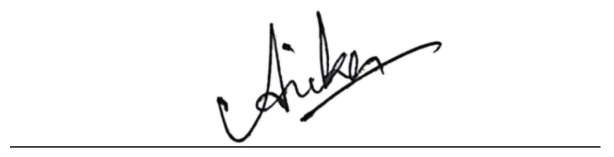

Anika Salsabil 17101498

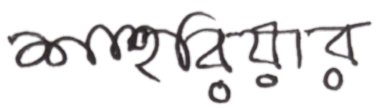

Mir Nafis Sharear Shopnil 17101423

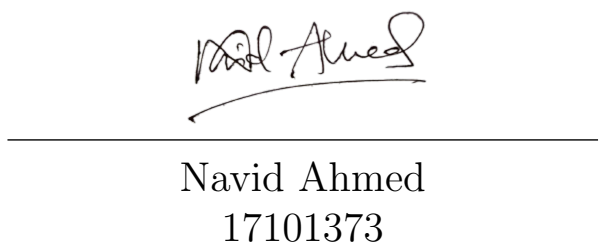




\section{Approval}

The thesis titled "Demystifying Black-box Learning Models of Rumor Detection from Social Media Posts" submitted by

1. Faiza Tafannum (17101063)

2. Mir Nafis Sharear Shopnil (17101423)

3. Anika Salsabil (17101498)

4. Navid Ahmed (17101373)

Of Summer, 2021 has been accepted as satisfactory in partial fulfillment of the requirement for the degree of B.Sc. in Computer Science on September 26, 2021.

\section{Examining Committee:}

Supervisor:

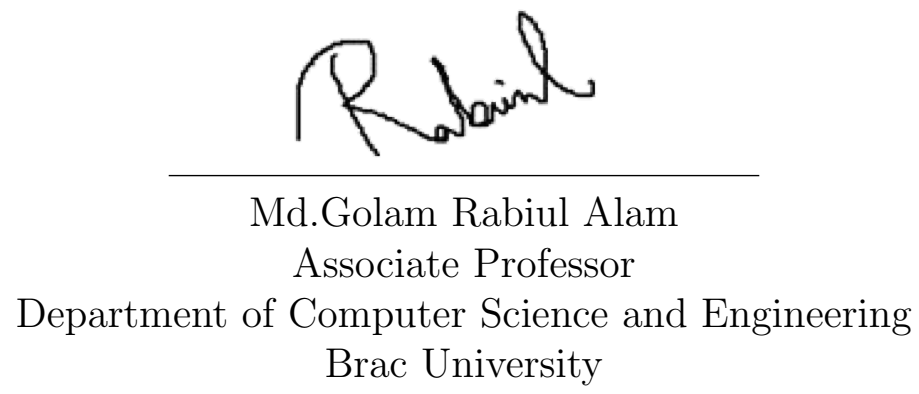

Co-supervisor:

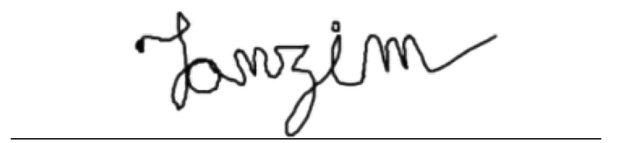

Md. Tanzim Reza

Lecturer

Department of Computer Science and Engineering

Brac University

Program Coordinator:

Md.Golam Rabiul Alam

Associate Professor

Department of Computer Science and Engineering Brac University 
Head of Department:

(Chairperson)

\author{
Ms.Sadia Hamid Kazi \\ Head of the Department \\ Department of Computer Science and Engineering \\ Brac University
}




\section{Abstract}

Social media and its users are vulnerable to the spread of rumors, therefore, protecting users from these rumors spread is extremely important. This research proposes a novel approach for rumor detection in social media that consists of multiple robust models: Support Vector Machine, XGBoost Classifier, Random Forest Classifier, Extra Tree Classifier, and Decision Tree Classifier. To evaluate more, we combine these five different machine learning models to build our own hybrid model. Then, we apply two deep learning models- Long-Short Term Memory (LSTM) and Bidirectional Encoder Representations from Transformers (BERT) and both show promising results with high accuracy. For evaluations, we are using two datasetsCOVID19 Fake News Dataset and Twitter15 and Twitter16- two publicly available datasets concatenated. The datasets contain posts from both Facebook and Twitter. We extract the textual part of source posts in vector representations and fit them into the models for predicting results and we evaluate the results. These artificial intelligence algorithms are often referred to as "Black-box" where data goes in the box and predictions come out of the box but what is happening inside the box frequently remains cloudy. Although there have been many inspired works for fake news detection, still the number of works regarding rumor detection lags behind and the models used in the existing works do not explain their decision-making process. But with explainable AI, the opaque process happening inside the black box can be explained. We use LIME to explain our models' predictions. We take models with higher accuracy and illustrate which feature of the data contributes the most for a post to be predicted as a rumor or a non-rumor by the models, thus, demystifying the black box learning models. Our hybrid model achieves an accuracy of $93.22 \%$ and $82.49 \%$, while LSTM provides 99.81\%, 98.41\% and BERT provides $99.62 \%$, $94.80 \%$ accuracy on the COVID-19, Twitter15 and Twitter16 datasets respectively.

Keywords: Social media; Rumor; Detection; Black box; Machine learning; Deep learning; Explainable; LIME; COVID-19; Classifier 


\section{Acknowledgement}

Firstly, all praise to the Great Allah for whom our thesis have been completed without any major interruption. Secondly, to our supervisor Dr. Md. Golam Rabiul Alam sir for his kind support and advice in our work. He helped us whenever we needed help.

Thirdly, to our co-supervisor Md. Tanzim Reza sir for his kind support and advice in our work. He also helped us whenever we needed help.

And finally to our parents without their throughout support it may not be possible. With their kind support and prayer we are now on the verge of our graduation. 


\section{Table of Contents}

Declaration $\quad$ i

Approval ii

Abstract $\quad$ iv

Acknowledgement $\quad$ v

Table of Contents vi

List of Figures viii

List of Tables $\quad$ x

Nomenclature xii

1 Introduction $\quad 1$

1.1 Research Problem . . . . . . . . . . . . . . . . . . . 2

1.2 Research Objective: . . . . . . . . . . . . . . . . . 4

2 Literature Review 5

2.1 Machine Learning . . . . . . . . . . . . . . . . . 5

2.1.1 Methods and Models . . . . . . . . . . . . . 5 5

2.2 Related Works . . . . . . . . . . . . . . . . . . 6

3 Methodology $\quad \mathbf{1 0}$

3.1 Data Collection: . . . . . . . . . . . . . . . . . . 10

3.2 Data Pre-processing . . . . . . . . . . . . . . . . . . . 10

3.3 Feature Extraction . . . . . . . . . . . . . . . . . . 12

3.3.1 Machine Learning Models . . . . . . . . . . . . . . . . 12

3.3.2 Deep Learning Models . . . . . . . . . . . . . . . . . . . . . . 13

3.4 Implementing Algorithms . . . . . . . . . . . . . . . . . . . . . 13

3.5 Visualizing Workflow . . . . . . . . . . . . . . . . . 21

4 Results and Analysis $\quad 22$

4.1 Results for Machine Learning Models . . . . . . . . . . . . . . . . . . 22

4.2 Results for Deep Learning Models . . . . . . . . . . . . . . . . . . . . . 26

4.3 Decision from Data . . . . . . . . . . . . . . . . . . . 29 
5 Explainable AI $\quad 30$

5.1 Squeezing LIME . . . . . . . . . . . . . . . . . . . . . . 30

5.1.1 LIME on Machine Learning Models . . . . . . . . . . . . . . 31

5.1 .2 LIME on LSTM . . . . . . . . . . . . . . . . . . . . . 32

6 Future Work Plan and Conclusion 35

6.1 Scopes in the Future . . . . . . . . . . . . . . . . . . 35

6.2 Conclusion . . . . . . . . . . . . . . . . . . . . 35

$\begin{array}{ll}\text { Bibliography } & 38\end{array}$ 


\section{List of Figures}

3.1 Covid-19 Fake News dataset . . . . . . . . . . . . . . . . . 11

3.2 Twitter 15 and Twitter 16 dataset . . . . . . . . . . . . . . . . 11

3.3 Dataset after pre-processing . . . . . . . . . . . . . . . 11

3.4 Covid-19 Fake News dataset . . . . . . . . . . . . . . . . . . . . . . . . . . . . . . . 12

3.5 Twitter 15 and Twitter 16 dataset . . . . . . . . . . . . . . . 12

3.6 Building the Hybrid Model . . . . . . . . . . . . . . . . . 15

3.7 LSTM Architecture . . . . . . . . . . . . . . . . . . . . . . . 18

3.8 Used LSTM Model . . . . . . . . . . . . . . . . . . . . . . . 18

3.9 BERT Architecture . . . . . . . . . . . . . . . . . . . . . . 19

3.10 Used BERT Model (one layer) . . . . . . . . . . . . . . . . . . 20

3.11 Workflow of XGBoost, SVC, RFC, ETC, DTC and Hybrid models . . 21

3.12 Workflow of LSTM and BERT models . . . . . . . . . . . . . . . 21

4.1 Con mat for XGBoost . . . . . . . . . . . . . . . . . . . 24

4.2 Con mat for SVM . . . . . . . . . . . . . . . . . . . . . . 24

4.3 Con mat for RFC . . . . . . . . . . . . . . . . . . . . 24

4.4 Con mat for ETC . . . . . . . . . . . . . . . . . . . . . . . . . . . . . . . . . . . 24

4.5 Con mat for DTC . . . . . . . . . . . . . . . . . . . . 24

4.6 Con mat for Hybrid . . . . . . . . . . . . . . . . . . . . . . . 24

4.7 Con mat for XGBoost . . . . . . . . . . . . . . . . 25

4.8 Con mat for SVM . . . . . . . . . . . . . . . . . . . . . 25

4.9 Con mat for RFC . . . . . . . . . . . . . . . . . . . . . . . . . . . . . . . . . . . . 25

4.10 Con mat for ETC . . . . . . . . . . . . . . . . . . . . . . . . . . . . . . . . . . . . 25

4.11 Con mat for DTC . . . . . . . . . . . . . . . . . . . . 26

4.12 Con mat for Hybrid . . . . . . . . . . . . . . . . . . . 26

4.13 Evaluation of LSTM on COVID-19 Dataset . . . . . . . . . . . . . 27

4.14 Evaluation of LSTM on Twitter 15 and Twitter 16 Dataset . . . . . . 27

4.15 Evaluation of BERT on COVID-19 Dataset. . . . . . . . . . . . 28

4.16 Evaluation of BERT on Twitter 15 and Twitter 16 Dataset . . . . . . 28

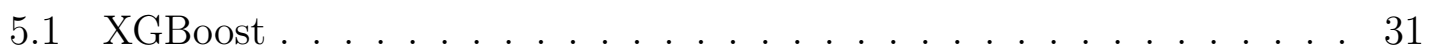

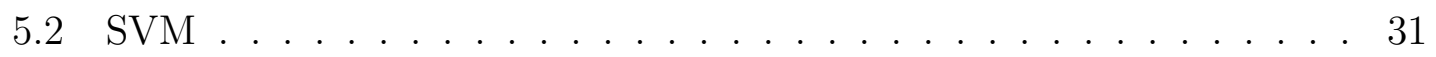

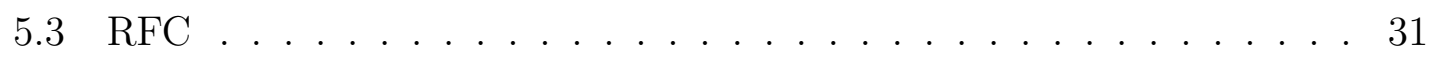

5.4 ETC . . . . . . . . . . . . . . . . . 31

5.5 Hybrid . . . . . . . . . . . . . . . . . . . 31

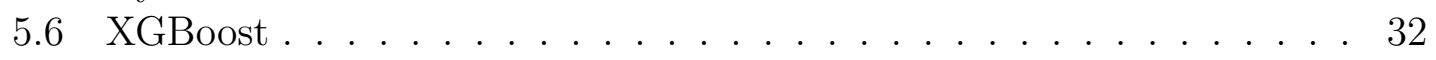

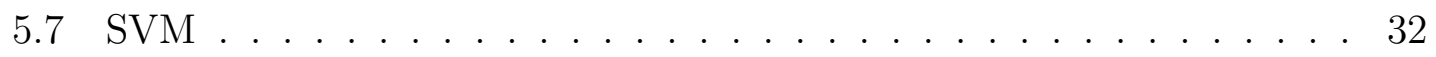

5.8 ETC . . . . . . . . . . . . . . . . . . . . . 32

5.9 Hybrid . . . . . . . . . . . . . . . . . . . . . . . 32 
5.10 LSTM's Explanation (COVID-19 Fake News dataset) . . . . . . . . . 33

5.11 LSTM's Explanation (concatenation of Twitter 15 and Twitter 16

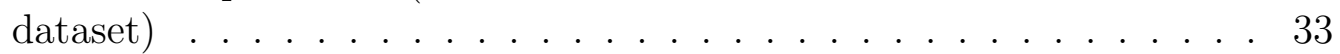




\section{List of Tables}

3.1 Hybrid Model Parameters . . . . . . . . . . . . . . . . . . . 17

4.1 Accuracy, precision, recall and F1 scores (COVID-19 Fake News Dataset) 23

4.2 Accuracy, precision, recall and F1 scores (concatenation of Twitter 15, Twitter 16 Datasets) . . . . . . . . . . . . . . 23

4.3 Accuracy scores (COVID-19 Fake News dataset) . . . . . . . . . . . 28

4.4 Accuracy scores (Twitter 15 and Twitter 16 dataset) . . . . . . . . 29 


\section{Nomenclature}

The next list describes several symbols \& abbreviation that will be later used within the body of the document

AI Artificial Intelligence

$A N N$ Artificial Neural Network

BERT Bidirectional Encoder Representations from Transformers

$B I-L S T M$ BI Directional Long-Short Term Memory

$B P E$ Byte Pair Encoding

CNN Convolutional Neural Network

COVID - 19 Corona Virus Disease 2019

$D L \quad$ Deep Learning

DTC Decision Tree Classifier

ETC Extra Tree Classifier

FP False Positive

GBM Gradient Boosting Machine

$H A S-B L S T M$ Hierarchical Social Attention Network-BI Directional Long-Short

Term Memory

$k N N$ The k-nearest Neighbors

LightGBM Light Gradient Boosting Machine

LIME Local Interpretable Model-agnostic Explanations

LSTM Long-Short Term Memory

$M L \quad$ Machine Learning

NLP Natural Language Processing

NLTK Natural Language Toolkit

$O C C$ One-Class Classification 
$R F C$ Random tree Classifier

$R N N$ Recurrent Neural Network

$R v N N$ Recursive Neural Network

SIR Susceptible Infective Removed

$S V M$ Support Vector Machine

$T F-I D F$ Term Frequency-Inverse Document Frequency

$T N$ True Negative

TP True Positive

$U R L$ Uniform Resource Locator

WHO World Health Organization

XGBOOST Extreme Gradient Boosting 


\section{Chapter 1}

\section{Introduction}

The rumor-spreading problem is one of the most severe obstacles communities have encountered for many years. In reality, it is an old topic that can destroy the credibility of a person or an organization, shake capital markets, and disrupt aid operations. These forms of knowledge have historically been circulated by word of mouth, newspapers, and radio. However, the development and rapid growth of online social networks have evolved this issue into a significant socio-political challenge in recent years due to the fast, quick, and broad distribution of online social network knowledge. It is tough to consult experts or authoritative organizations to stop rumors from spreading on social media. Rumor generally spreads very fast and reaches many users before experts or authoritative organizations can conclude. Therefore, it is essential to automatically detect rumors at the early stages of their development, so the Rumor's negative impact can be minimized. The news may be posted on Twitter without sufficient supervision or checking, resulting in unconfirmed and unverified claims being distributed. Public panic and social damage may be triggered by such false news or rumors. For instance, a Twitter account called "Associated Press" falsely reported on April 23, 2013, that two bombings happened in the White House and that President Obama was wounded in the attack. A few minutes later, the White House and "Associated Press" dismissed the news and announced the "Associated Press" account's breach Chen et al.[1] Similarly, During the 2016 US presidential election [2], there were plenty of myths and false reports, such as "Trump won both the popular vote and the Electoral College."

Vosoughi[3] cited that fake news spreads much quicker than facts. The chance for People to spread information containing fear, confusion, and credulity is high [4]. The rapid spread of misinformation or false news and rumor or non-verified information may have a severe negative impact on people and culture, such rumors can influence the understanding of the news ecosystem's credibility, leading to a general skepticism regarding all news on Twitter; rumors can alter the way people believe or react to actual information and news.

Researchers use large-scale statistics and computer tools to monitor the phenomena of Rumor spreading because of the frequency and pace of rumors on social networks. A better approach for rumor identification is required to resolve the above issues, considering different environmental factors. We examine various rumor topics, characteristics, ML and DL approaches in the first step; then we try to explain the 
models' prediction using Explainable AI. In order to accomplish this purpose, we propose to experiment with ML and DL algorithms.

\subsection{Research Problem}

As more people are joining social media every day, Rumor spreading is increasing. According to [5], the number of social media users by the end of 2023 will be 3.96 billion. Having a massive increase in the number of devices by 2025 underlines the question of what sort of misinformation these users will be affected by misinformation. A decision-maker, power network operator, Internet service provider, or target of a malicious rumor would like to determine the danger source as soon as possible in all of these cases and then quarantine the impact.

Rumor detection has been a concern of importance to the social computing community. The primary objective of rumor identification is to automatically, reliably, and classify rumors on online social networks promptly. With this in mind, it is fascinating to learn about identifying and discouraging the dissemination of rumors.

There are several descriptions of rumors in literature. Rumor is referred to as a piece of inaccurate information. Some scholars defined a rumor as, "An unconfirmed statement or fake news that often carries malicious information that can be created intentionally or unintentionally". Rumors have also been stated as "Unverified and instrumentally relevant information statements in circulation that arise in contexts of ambiguity, danger or potential threat, and that function to help people make sense and manage risk". Rumor is stated as "a currently circulating story or report of uncertain or doubtful truth" ("rumor," n.d.) by Oxford dictionary.

Below are several notable aspects of rumor transmission on various social networking sites:

Temporary properties of the Rumor spread: rumors will only grow for a brief amount of time according to various social psychological hypotheses and are often purposely spread to conceal the lack of news from the institutional outlets [6]. Such data were classified after questioning as a rumor. The systemic features of rumors spread: a rumor study claimed that dense network systems are less prone to social fragmentation. Kwon[6] quoted a report on gossips that indicates that in sparse systems, gossips disperse more widely.

Linguistic features of the Rumor spread: a report on laboratory interviews suggested that rumors are likely to be dominated by such forms of phrases as fear, confusion, and credulity [6].

Rumors from these points of view are a primary type of disinformation that is distinguished by two characteristics. First, the rumors are claims without precise proof requirements. Second, through widespread social distribution, rumors gain their power, Berinsky [1] 
In recent years there are multiple studies conducts in efforts of rumor detection. Most previous studies use machine learning to detect rumors. In most of these works, the common problem is related to feature extraction from a considered dataset. It takes a considerable portion-time and effort to manually extract features, with little reliability in identifying rumors for most of these works. Deep neural networks have recently been suggested to simplify the extraction of properties and provide an excellent capacity to learn abstract representation [7]. Most automated rumor detection systems regard the issue of rumor detection as a binary classification activity, according to Cao [8], and they can be divided into one of the three perspectives below.

i) Machine learning (in particular, ML-based on hand-crafted features): to explain the spread of rumors in high-dimensional space, they add hand-crafted features. For these methods, function engineering is a critical necessity. Moreover, these methods abstract features from both textual and visual information based on which the hyperplane is segregated by a classifier ( [9]-[10]).

ii) Networking model: In contrast to the ML model, multiple heterogeneous systemic social networking characteristics (such as the number of followers, comment content, timestamp, etc.) are used to test network legitimacy through the networking paradigm along with graph-based optimization approaches ( [10]-[11].

iii) Deep learning (DL) paradigm: this group's strategies are used automatically to train and combine multi-modal functions.

Like the ML paradigm, this paradigm is focused on data learning. However, unlike the ML paradigm's handcrafted-features-based approach, the DL paradigm does not require any feature engineering. During the training phase, the classifier discovers and acquires the appropriate feature. There are many benefits to the DL paradigm, including a significant increase in efficiency and removing the tedious extraction process.

It is more advantageous for mathematical, numerical, and representative problems using an ensemble approach rather than searching for a single highest-performing algorithm model, stated by Dietterich [12]. Furthermore, observational findings in several previous papers have verified that high uncertainty, volatility, and bias [13]-[14] can be minimized with the use of the ensemble approach. There have been several approaches to using ensemble solutions to identify rumors in order to understand these advantages. An ensemble learning methodology incorporating techniques such as data sampling (i.e., arbitrary oversampling, arbitrary under-sampling, and fuzzybased oversampling) was introduced by Liu [15]. However, their primary use is spam identification, and various ML models were not regarded.

This research proposes various machine learning and deep learning models to detect rumors and non-rumors. With the help of these, this research will try to detect rumors and quarantine the effect quickly.

Therefore, the question that arises is that how successfully the proposed algorithms 
can detect rumors in social media. This research will answer the risen question by exploring, implementing and explaining algorithms.

\subsection{Research Objective:}

We aim to develop a rumor detection system for detecting both rumors and non rumors using multiple models: Support Vector Machine (SVM), XGBoost Classifier, Random Forest Classifier, Extra Tree Classifier, and Decision Tree Classifier. Moreover, to evaluate more, we use these five different machine learning models to build a hybrid ensemble model. Then, we apply two deep learning models- Long-Short Term Memory (LSTM) and Bidirectional Encoder Representations from Transformers (BERT). The, we use LIME to explain our models' predictions. Usually, models will collect datasets and send them for further processing via gateways to an evaluation system. Data sent into the models will be detected in the evaluation layer in the model. The objectives are:

i. To deeply understand Rumor and how it spreads.

ii. To deeply understand rumor detection techniques.

iii. To apply and evaluate the ML models.

iv. To apply and evaluate the DL models.

v. To explain the models' predictions using explainable AI.

vi. Understanding the black boxes better.

vii. To offer recommendations on improving the model for future work. 


\section{Chapter 2}

\section{Literature Review}

The current world has a crowd solely dependent on the Internet that leads their learning sources skewed towards social media platforms. Thus, the massive crowd shares information from platforms to platforms that convert into rumors. Most people tend to capture information from these platforms without verifying the facts, unlike a few percentages of people who check the validity of information/news from other sources. Several research groups had been researching the influence and impact of misleading rumors and rumor detection capabilities.

\subsection{Machine Learning}

Artificial Neural Networks (ANNs) structure is influenced by the way biological nervous systems work as "neural" part of the name suggests. ANN is subset of ML. Neural networks include several layers- input, output layers and a hidden layer comprising units that transform the input into something that the output layer can use. There are many interconnected process to make up one computational system that tries to solve a problem of a large dataset through learning intensively. Deep learning is a form of machine learning and a subset of ANN. It is also known as deep structured learning where the adjective "deep" comes from the use of multiple layers in the network. The hierarchy concepts help the computer to build more complicated concepts from the simpler ones and the graph is many layers deep.

\subsubsection{Methods and Models}

There are different types of ML algorithms that include Linear Regression, Logistic Regression, Decision Tree, SVM, Naive Bayes, kNN, K-Means, Random Forest, Dimensionality Reduction Algorithms, Gradient Boosting algorithms like GBM, XGBoost, LightGBM, CatBoost. Many DL algorithm's like different types of RNN, LSTMS (Vanilla, Bi-Directional, Pyramid, etc.), BERT, RvNN, etc. LIAR dataset, PHEME dataset, Twitter dataset, news articles, and other Rumor tracking websites are used to collect datasets. 


\section{$2.2 \quad$ Related Works}

In the past, to detect rumors and non-rumors and improve the techniques of detecting rumors on social networking sites, different models, frameworks, and approaches on various datasets have been proposed. For instance, according to [16], for rumor detection, mostly RNN architecture is preferred over pure CNN or RNN-CNN, CNN-RNN architectures.

We collected some works from IEEE Explore, Springer Link, ACM Digital Library, and Google Scholar- these digital libraries and reviewed them. These papers have been hand-picked from a vast amount of research conducted in the past few years on rumor detection techniques. We try to present some limitations of those researches within our knowledge to illustrate how rumor detection has yet many challenges to face.

During the COVID-19 pandemic, rumors have been spreading fast. Some research has been conducted using machine learning techniques to analyze the far-reaching spread of both accurate and inaccurate information and sentiments of the public about the information. By conducting these researches, the authors found people's emotions regarding the topics. According to [17], contrary to analyzing infodemics, the authors of the paper focus on how COVID-19 rumors scatter, how people get engaged and what emotions are invoked. Mythbusters by WHO have been used, and the authors matched keywords to find out tweets about COVID-19 myths using Twitter API. Firstly, the myths are classified into five categories to find out the primary topic of Rumor, and the tweets are fed to the SIR epidemic model. After that, the model is used to calculate the primary reproduction number (R0) for each category of myths. From this, the authors found out which category of myths spread more quickly and rose faster. To approximate R0, the negative log estimates the model parameters, and the range of parameters is estimated via the Nelder-Mead algorithm.

Another research [18] proposes an end-to-end model for multi-modal fake news detection called BERT-based domain adaptation neural network (BDANN). A multimodal feature extractor, a domain classifier, and a fake news detector are the three main modules of the BDANN feature extractor that retrieve the multi-modal features, which are concatenated and provided to the domain classifier. The detector is used to distinguish between real and fake news.

[19] It aims to detect and eliminate rumors from online social networks regarding the pandemic. The authors decide that if $\mathrm{R} 0>1$, then the Rumor will continue spreading, otherwise it will not. This model uses some mathematical approaches and utilizes differential equations to block users to stop spreading rumors. They use the Jacobian matrix, eigenvalues of the matrix, Lyapunov function, etc. We found that the limitation in this rumor blocking model, it targets and blocks the Rumor only when the mathematical graph reaches a point. If it is less than 1 , it does not block the spread. This model cannot ensure that a verified user will not propagate unwanted messages in the network. They may be victimized by other unauthenticated users and may be moved into the category of rumor spreader. 
[20] Introduces speech act for Twitter which is a new tweet act classifier that evaluates the content and intent of tweets. They described BERT-Caps to deliver pre-trained deep bidirectional representations of massive unlabeled data. The suggested model outperformed many robust baselines and state-of-the-art methods and achieved a benchmark accuracy of 77.52 percent. The proposed approach learns features about voice acts and Twitter by exploiting the joint optimization of the pre-trained BERT model and capsule layer.

[21] Uses SI model to detect the source of a rumor proposing rumor centrality. Again, [22] proposes a SIR-based model, which is implemented on a tree-like network, and the reverse-infection algorithm produces an estimator, and the result that they get is closer to the actual source. However, according to [23], the simulation of these systems shows that they have a low rumor source detection rate.

To address this issue of Rumor spreading, [24] proposes a method structural and graphical properties of a tweet's propagation and the texts as a base to tell how a specific class of news spreads and also the characteristics of users involved in spreading that news. They used Twitter 15 and Twitter 16 benchmark datasets. The results for Rumor and fake detection are promising. For this, they use LSTM networks and RoBERTa based vector representations. Textual features from source tweets, profile features were used and modeled into a graph-structured propagation path in order to perform supervised graphical classification using graphical convolution networks.

[25] Proposes an RNN-based deep attention model which works on sequential tweets on the microblogging site called Tweeter for rumor identification and can deal with duplicate data. From the sequential posts, the model learns what the posts represent that cannot be identified just by looking at the sequential posts. It also finds distinct features of the posts. Then, with time, it constructs a concealed characterization that captures the contextual dissimilarity of the relevant tweets. We felt that the issue here is that large datasets are needed to feed the RNN based model, but it can often be challenging to get that kind of data actually relevant to the purpose of the model.

According to [26], traditionally, rumors are classified with a binary approach - Rumor or non-rumor. The researchers propose One-Class Classification (OCC), where they do not need the non-rumor data. The feature is extracted from tweets, and then they apply seven OCC's from their diverse paradigms and compare how they work. Here, they mainly use textual features. As claimed by [15], employing DL can give fruitful results while dealing with visual formats such as videos and images.

[27] Classifies all of the posts that are related to the 2016 US presidential election as rumor events. This research offers a new hierarchical social attention network (HSA-BLSTM). The rumor events are broken down into many sub-events, which are then used as input to a hierarchical Bi-LSTM network. This paper states that they use social traits and linguistic information. For accuracy, a new network with social properties is used. A flaw of this model is that it only considers the early stages of rumor dissemination. 
[28] It aims to eliminate fake news. Twitter and Weibo datasets are used. Here, to give knowledge about image characteristics, VGG-19 pre-trained images are used. BERT-based version is utilized, which includes 12 levels of encoding. The sequence of words is fed as input, and they are placed in front of a sequence of tokens. The remaining output layers are the features derived from the module's second output layer. A multi-modal fusion module, which fuses multiple representations to get a news feature vector, is the last sub-module. The fake news classifier combines multiple news detection, fake news detection, and multiple text and image to detect false news.

[29] Claims using a unique Recurrent Neural Network with an att-RNN, and the result is a proposed end-to-end network obtained via an LSTM (Long-Short Term Memory) network. The outputs of the LSTM are used for classification. For merging features from the text, image, and social context, end-to-end RNN with attention mechanism is presented. The model is tested on two multimedia datasets, and the RNN delivers excellent performance. Social environment and text data are merged with an LSTM. This paper states that we have already trained a deep CNN to derive visual data. Joint social environment and text data are combined with this visual data. Extensive experiments on the datasets show efficiency in rumor detection.

According to [30], a model is proposed to detect rumor tweets inspired by many efficient attention-based strategies. Here, the input sequence is weighted and averaged by the attention layer to get relevant information. LSTM + Attention (Text) and LSTM + Attention (Text) (hybrid features) - these two distinct models are used to detect rumors. Both are deep learning-based models. Both models have F1 scores in the range of 0.85-0.87 and 0.86- 0.89 .

[31] It aims to detect medical rumors on Twitter using machine learning. A classifier is built in this project that uses assertion; assertions are speech acts that can be labeled as either true or false. Assertions are fed to the classifier, then ranked based on the type of source-verified or unverified. Context and sentiment are used as a base here to compare trusted tweets with non-verified sources. If there is dissimilarity, then the tweets go through ML algorithms that analyze specific features with an accuracy of $90 \%$.

[32] proposes a multi-task learning approach for rumor detection and stance classification tasks. The model contains multiple layers like the stance detection layer, the rumor verification layer. The model takes the user credibility into the layer of rumor detection and deploys an attention mechanism in the process of rumor detection. One of the datasets used in this research had no stance labels, so to get stance labels, a stance detection algorithm is used. The task was challenging and easier for source tweets than for replying tweets, meaning that there were confliction instance labels. An analysis is run to find similar posts to make the labels more comfortable. The similarity is calculated. There are yet some limitations of the model proposed.

As stated in [33], this paper proposes an Attention-Residual network merged with CNN (ARC). This model represents a contextual feature for which a data encoding 
model is built. Content features are essential clues for rumor detection. Then, a residual framework is proposed, which is based on fine-tuned attention mechanism. Then, a convolution neural network or CNN is applied to select some features and components. This model is applied to two datasets. The drawback is that CNN is significantly slower and takes time to compute.

[34] Views rumor detection task as anomaly detection task and uses neural networks. An unsupervised learning model is proposed that combines RNN and autoencoders to detect rumors from social media platforms or microblogs. For feature extraction, Weibo's set, Weibo's feature, Weibo's comments are extracted, and features from comments are extracted. The authors observe temporal behaviors because of the difference in the propagation of Rumor and non-rumors. Between different time slots, there are higher-level feature interactions, and in order to capture it, a multiple hidden layer structure of RNN is developed. The values of the last hidden layer help to calculate the output. Then, the autoencoder is combined with RNN for rumor detection.

The study in [35] shows how to train temporal representations of sequential postings for rumor identification using a deep attention model based on recurrent neural networks (RNNs). Extensive tests using real data from social media platforms show that the model surpasses state-of-the-art baselines in recognizing rumors faster and more correctly than rivals. The authors proposed a novel deep attention based recurrent recurrentneural network (RNN) for early detection on rumors, namely CallAtRumors (Call Attention to Rumors). The RNN with soft attention mechanism automatically learns latent representations by feed-forwarding each input weighted by attention weights. An additional hidden layer with sigmoid activation using the learned latent representations to classify whether this event is a rumor or not. Rumor detection on social media is time-sensitive because it is hard to eliminate the vicious impact in its late period of diffusion. CallAtRumors is based on soft attention mechanism to automatically carry out early rumor detection.

As we building a model that features multi-lingual rumor detection could be interesting, we would like to mention rumor detection for Persian tweets [36]. In this paper, the authors collect and label a dataset of Persian Rumor and non-rumor tweets. Some structural features have been used from tweets and user characteristics. Firstly, Rumor is detected applying structural features, and the performance of the classifier is measured, which yields $70 \%$ precision. Then, Rumor is detected applying structural and content-based features, and again, the performance of the classifier is measured, which yields $80 \%$ precision. Lastly, the most discriminative features are introduced and analyzed. Although this paper tackles an uninvestigated problem of rumor detection, a higher rate of precision is yet to be achieved.

All the researched that have been conducted so far are inspiring, yet the decision making process of the models remain unknown, therefore, we try to make our models interpretable to make a contribution towards making the process of rumor detection explainable. 


\section{Chapter 3}

\section{Methodology}

\subsection{Data Collection:}

People are actively using Twitter, Facebook, and other social media sites regularly all around the world. So, the proposed rumor detection models aim to detect rumors and non-rumors from the data from social media sites. We collected a dataset from the "Constraint@AAAI2021 - COVID19 Fake News Detection" competition that is domain-specific and contains social media posts during the COVID-19 pandemic. There are approximately 8560 posts. All the posts in the dataset are both Rumor and non-rumor. The "tweet" column comprises posts, either Rumor or non-rumor, collected from popular social media sites like Twitter and Facebook. The "label" column labels the tweets as real or fake. For example, "Alfalfa is the only cure for COVID-19." is a post that is a rumor and labeled as fake. On the other hand, "As of today, eight states are reporting racial demographics for COVID cases: CT IL MI MN NC NJ SC VA and five states are reporting racial demographics for COVID deaths: CT IL LA MN NC," is a post which is not a rumor, hence labeled as real. We have also used the publicly available dataset named Twitter15 and Twitter 16 released by [35]. These datasets are not domain-specific and they contain posts about a wide variety of topics. We have labeled all rumor events in the datasets as "rumor" and labels of non-rumor events have been kept the same- "non-rumor". We have dropped rows containing unverified information. We concatenated the datasets for our usage and both the datasets together contain approximately 1736 posts from both Facebook and Twitter. An example is, "a traditional beer that authorities believe was poisoned with crocodile bile has killed 56 people in Mozambique: URL" is labeled as rumor and "former 3 doors down guitarist Matt Roberts has died at age 38 , according to his father. URL URL" is labeled as non-rumor. We specifically chose two different types of datasets (domain-specific and not domain-specific) to see how the models perform on each dataset.

Highlighted words in the word clouds below illustrate the topics in each dataset.

\subsection{Data Pre-processing}

All the posts collected from social media are in raw form in the dataset, which is not useable to train machine learning algorithms. Data pre-processing is a way of 


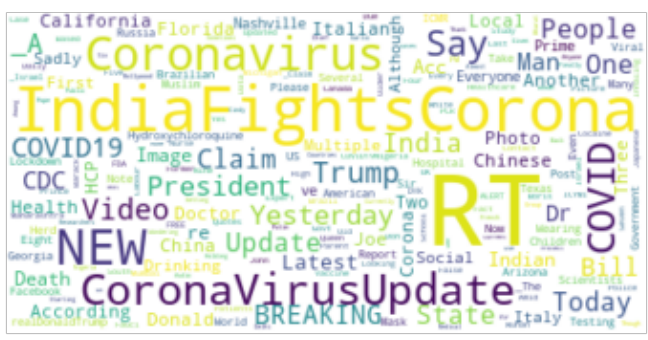

Figure 3.1: Covid-19 Fake News dataset

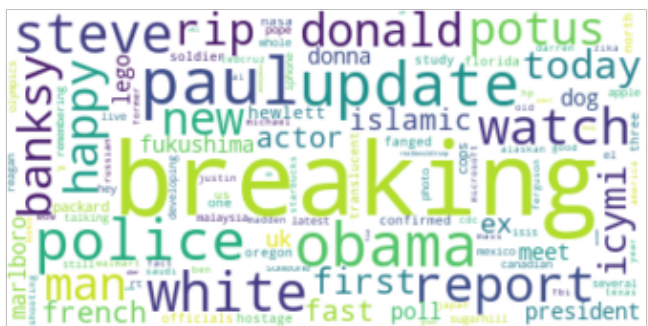

Figure 3.2: Twitter 15 and Twitter 16 dataset

getting raw data ready for Machine Learning algorithms, i.e., by rescaling, one-hot encoding, label encoding, lemmatizing, etc. We used the Python programming language for coding. We used NLTK, or Natural Language Toolkit, a Python package for Natural Language Processing. Firstly, we removed all links from the posts and replaced those with empty strings. Then, we removed emoticons, symbols, pictographs because these are unnecessary for analyzing the text data. Punctuations, English stop words, and all non-alphanumeric characters have been removed as well. Secondly, we tokenized the social media posts. Tokenization is a process in NLP by which a raw text is broken into smaller parts called tokens. After tokenization, we applied lemmatization, by which we can generate the root or base form of the words in the text data. We used WordNet corpus to produce lemma. After that, we transformed the non-rumors to binary value "1" and rumors to binary "0" using Python's built-in map() function. Below is the resulting dataset.

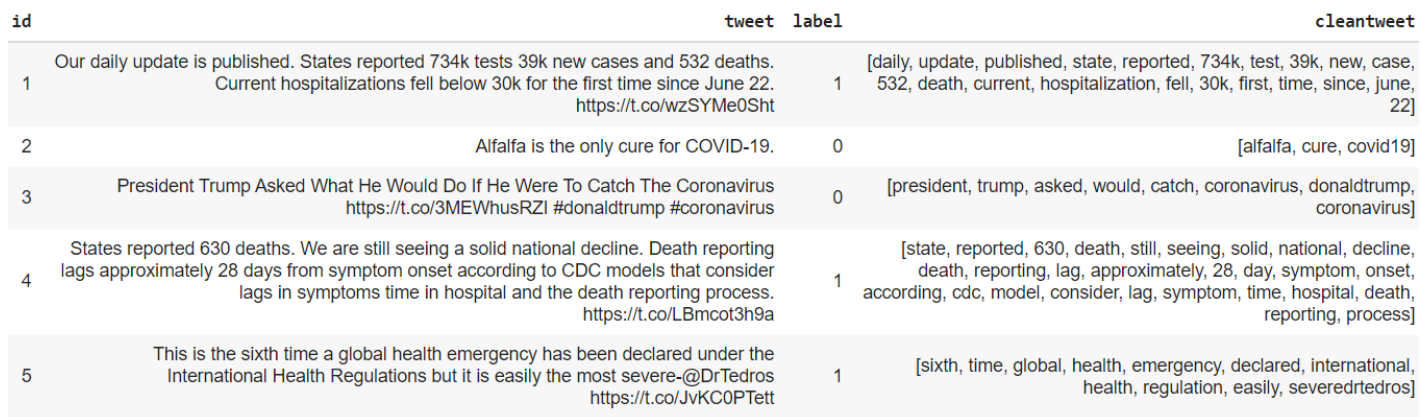

Figure 3.3: Dataset after pre-processing 
The proportion of rumor and non-rumor posts after data pre-processing can be visualized from the figures.

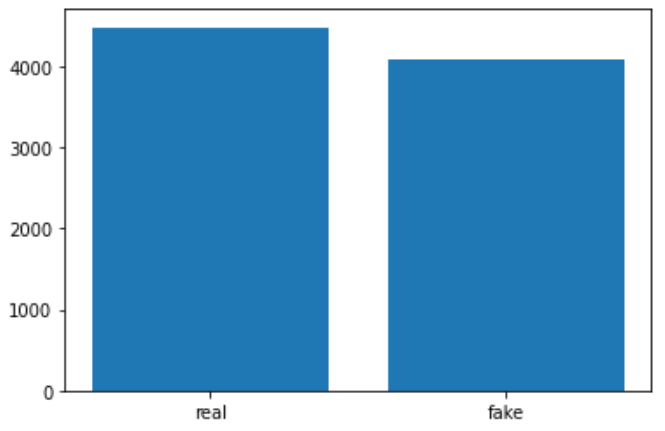

Figure 3.4: Covid-19 Fake News dataset

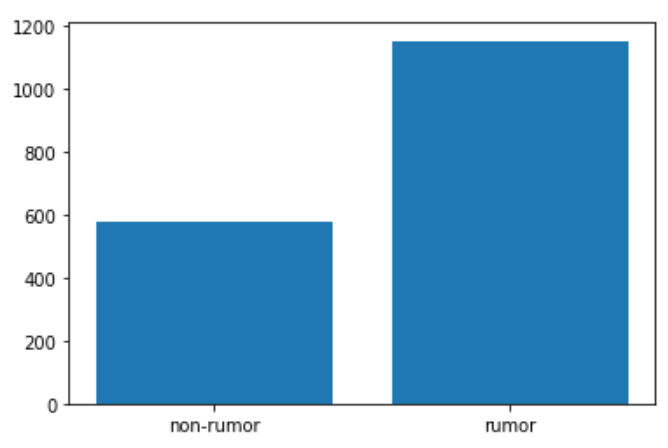

Figure 3.5: Twitter 15 and Twitter 16 dataset

\subsection{Feature Extraction}

\subsubsection{Machine Learning Models}

For the machine learning models, TF-IDF which stands for Term Frequency - Inverse Document Frequency has been used for feature extraction from texts as it is based on word statistics. TF-IDF uses two statistical methods, the first is Term Frequency, and the other is Inverse Document Frequency. TF-IDF Vectorization creates a document term matrix where the columns are individual unique words like count vectorizer and the cells contain weight which signifies how important a word is for an individual text message. The weight is calculated using the formula below,

$$
w_{i, j}=t f_{i, j} * \log \left(\frac{N}{d f_{i}}\right)
$$

Here, term frequency or $\mathrm{tf}_{i j}$ the number of times term $\mathrm{i}$ occurs in $\mathrm{j}$ divided by the total number of terms in $\mathrm{j}, \mathrm{N}$ is the total number of documents in the corpus, and $\mathrm{df}_{\mathrm{i}}$ is the number of documents containing the term $\mathrm{i}$. In this case, a single tweet or a post is a document. Thus, the amount of information a word provides is measured by inverse document frequency. This formula provides numeric values for the entire document, and of course, in this case, for each tweet. For example, we take a tweet, $\mathrm{j}=$ "Squeezing hot lemon water cures coronavirus infection." from our dataset. Let, term, $\mathrm{i}=$ cure So, term frequency, $\mathrm{tf}_{\text {cure }} \mathrm{j}=1 / 7=0.14$ Total number of documents, $\mathrm{N}=8560$ : [This dataset contains 8560 posts Document frequency, $\mathrm{df}_{\text {cure }}=249$ [The word 'cure' is present in 249 posts ] Therefore, weight of the word, $\mathrm{w}_{\text {cure. }} \mathrm{i}=\mathrm{tf}_{\text {cure } j} * \log \left(\frac{\mathrm{N}}{\mathrm{df}_{\text {cure }}}\right)$

$$
\begin{aligned}
& =0.14 * \log \left(\frac{8560}{249}\right) \\
& =0.2
\end{aligned}
$$

We applied TF-IDF Vectorization on the clean tweet that we had gotten after text data pre-processing. 


\subsubsection{Deep Learning Models}

Both LSTM and BERT use word embedding for feature extraction. LSTM uses pre trained word embedding while BERT tokenizes using BPE n-gram techniques. Then, it learns the embedding with the encoder and decoder jointly.

\subsection{Implementing Algorithms}

An artificial intelligence system conducts its task by Machine Learning algorithms, predicting output values from some given input data. A primary process of machine learning algorithms is classification. Classification algorithms are Supervised Learning algorithms. We have used XGBoost Classifier, Support Vector Machine, Random Forest Classifier, Decision Tree Classifier, Extra Tree Classifier, and the hybrid of all these models where we have defined each model five times with different hyper parameters that resulted in a combination of twenty five models. Then we used Max Voting Classifier, where the class that has been predicted most by the underlying models, will be the class predicted by the hybrid model. We trained our dataset to classify discrete values such as rumor or non-rumor and predict the output. To do so, we have split the COVID-19 dataset of 8560 posts and Twitter 15 and Twitter 16 datasets into a train $(75 \%)$ and test sizes $(25 \%)$ for the machine learning models and a train $(80 \%)$ and test sizes $(20 \%)$ for the deep learning models.

We are going to discuss how our used models work below:

\section{XGBoost:}

In recent years, the XGBoost algorithm has dominated the machine learning community. It is a gradient boosted decision tree implementation optimized for speed and performance. The XGBoost implementation incorporates elements from ScikitLearn and $\mathrm{R}$, as well as novel features such as regularization. XGBoost model supports three types of gradient boosting:

i) Gradient Boosting,

ii) Stochastic Gradient Boosting

iii) Regularized Gradient Boosting

The algorithm used by XGBoost is the gradient boosting decision tree algorithm. Boosting is an ensemble strategy that involves adding new models to old models to fix inaccuracies. Models are added in a logical order until there are no more improvements to be made.

Some of the parameters that we use in $\mathrm{XGBoost}$ are learning rate $=0.15$; maximum depth $=8$ which is the maximum depth of a tree and we can control the over-fitting problem with it as higher depth will allow model to learn relations very specific to a particular sample; number of estimators $=300$ which allows XGBoost to use 300 trees as base learner; number of threads $=-1$; silent=True which will not allow any running messages to be printed; subsample $=0.9$ which will somewhat turn the algorithm to be more conservative and prevent overfitting. XGBoost bases the convex loss function on the difference between the expected and target outputs and combines it with 
a penalty term for model complexity so that $\mathrm{s}$ regularized objective function can be minimized. The training is done in repeatedly adding new trees which can predict the residuals or errors of the previous trees, The trees are then integrated with the previous trees to get the final prediction. Gradient boosting uses a gradient descent approach to minimize loss when adding new models.

\section{Support Vector Machine (SVM):}

Support Vector Machine or SVM is one of the popular models used in Machine Learning. SVM uses classification algorithms for two-label classification issues. We give SVM sets of labeled training data and it then categorizes new text for each category. When SVM is fitted with data with two different labels, it takes those data points and creates a hyperplane (a two-dimensional line) that separates the labels. This line is referred to as the decision boundary. Everything on one side is categorized as the same label, while everything on the other side is categorized as a different label. The optimum hyperplane that maximizes the margins from both labels is chosen by SVM. The hyperplane with the most significant distance to the nearest element of each label is, in essence, the largest.

Some of the hyperparameters used in SVM are number of estimators $=300$ which allows the algorithm to use 300 trees as base learner; kernel="linear", $\mathrm{C}=1.0$ which acts like a regularization parameter in the SVM and for this value of $\mathrm{C}$, a larger margin will be accepted resulting in a decision function which is simpler but at the cost of training accuracy; random state $=27$; probability $=$ True. These hyperparameters enable to get higher accuracy in the model. The kernel is usually linear. Therefore we get a linear classifier. When using SVM for Natural Language Classification, the text must first be translated into a vector of integers. In non-linear data, SVM can work without the actual vectors, relying instead on the dot products between them. We have utilized TF-IDF to calculate frequencies in a more complex manner.

\section{Random Forest Classifier:}

Random Forest is built from decision tree algorithms and can solve classification problems like ours. Root nodes and segregating nodes are established randomly in random forest. As the name suggests the 'forest' which is generated by the random forest algorithm is trained through bootstrap aggregating or bagging. It utilizes ensemble learning like XGBoost. The outcomes are established based on what the decision tree predicts and it can lower overfitting and increase precision. Although random forest can give a reasonable prediction result without hyperparameter tuning, the hyperparameters we used are number of estimators $=300$ which allows the algorithm to use 300 trees as base learner; maximum depth $=300$ which is the maximum depth of a tree and random state $=27$. Every tree in random forest has a root node, decision nodes, and leaf nodes. The leaf node represents the final output produced by a certain decision tree. The final output is selected by the majority-voting system. In this case, the output that most of the decision trees choose becomes the final output of the rain forest system.

\section{Extra Tree Classifier:}

Extra Tree Classifier is similar to RFC and also known Extremely Randomized Trees. It is called extremely randomized tree is because the features and splits are randomly selected. ETC is less computationally expensive than RFC as the splits 
are chosen randomly. The randomization reduces overfitting. Another feature is that it does not bootstrap observations. ETC is based on decision trees and is an ensemble learner. The hyperparameters we used are number of estimators $=300$ which allows the algorithm to use 300 trees as base learner; maximum depth $=300$ which is the maximum depth of a tree and random state $=27$.

\section{Decision Tree Classifier:}

Decision Tree is preferred for solving classification problems but it can also be used for regression problems. The classifier is tree-structured with internal nodes, branches and leaf nodes. Dataset features are represented by internal nodes, the decision rules are represented by branches and the outcome is represented by each leaf node. In a decision tree, decision nodes have many branches and these nodes are used to make a decision, whereas the output of those decisions is the leaf nodes and they do not contain any more branches. The decisions are performed based on the features of the datasets. The hyperparameters we used are maximum depth of a tree $=300$, criterion $=$ gini which measures the quality of a split. For the Gini impurity, the supported criteria are "gini". We use "entropy" for the information gain in some of the DTC models in our hybrid model. Splitter is set to "best" by default which is the strategy to choose the split.

\section{Hybrid model:}

We use these five different machine learning models discussed above to build a hybrid ensemble model. We have defined each model five times with different hyper parameters that resulted in a combination of twenty five models.

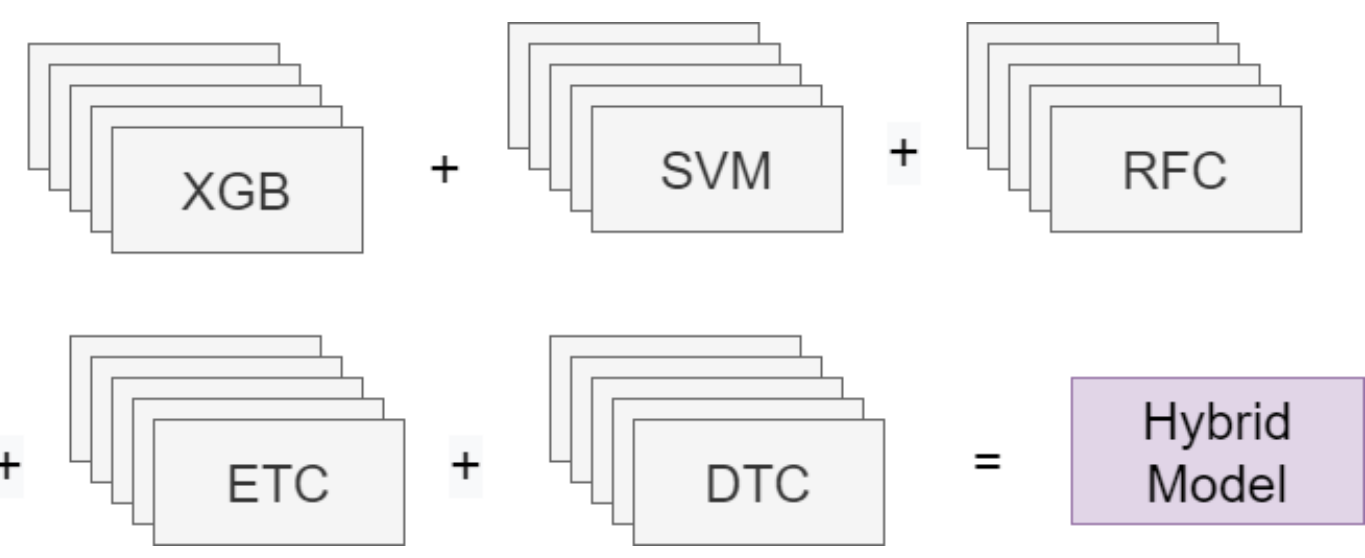

Figure 3.6: Building the Hybrid Model 
Then, we use Max Voting Classifier, where the class that has been predicted most by the underlying models, will be the class predicted by the hybrid model. In max-voting, each base model makes a prediction and votes for each sample. The hyperparameters used for each model is given in the table.

\begin{tabular}{|c|c|c|}
\hline Classifier & Model Number & Parameters \\
\hline Support Vector Classifier & Model 01 & $\begin{array}{l}\text { kernel="linear", } \mathrm{C}=1.0 \text {, random state }=27 \text {, } \\
\text { probability }=\text { True }\end{array}$ \\
\hline Support Vector Classifier & Model 02 & $\begin{array}{l}\text { kernel="sigmoid", } C=1.0 \text {, random state }=27 \text {, } \\
\text { probability }=\text { True }\end{array}$ \\
\hline Support Vector Classifier & Model 03 & $\begin{array}{l}\text { kernel="poly", } \mathrm{C}=1.0 \text {, random state }=27 \text {, } \\
\text { probability =True }\end{array}$ \\
\hline Support Vector Classifier & Model 04 & $\begin{array}{l}\text { kernel="sigmoid", } C=1.0 \text {, random state }=27 \text {, } \\
\text { probability }=\text { True }\end{array}$ \\
\hline Support Vector Classifier & Model 05 & $\begin{array}{l}\text { kernel="rbf", } C=1.0, \text { random state }=27, \\
\text { probability }=\text { True }\end{array}$ \\
\hline XGBoost & Model 06 & $\begin{array}{l}\text { learning rate }=0.15, \text { max depth }=8, \\
\text { number of estimators }=300, \\
\text { number of threads }=-1, \\
\text { silent }=\text { True, subsample }=0.9\end{array}$ \\
\hline XGBoost & Model 07 & $\begin{array}{l}\text { learning rate }=0.2, \text { max depth }=7 \text {, } \\
\text { number of estimators }=200, \\
\text { number of threads }=-1 \text {, } \\
\text { silent=True, subsample }=0.9\end{array}$ \\
\hline XGBoost & Model 08 & $\begin{array}{l}\text { learning rate }=0.25, \text { maximum depth }=8 \text {, } \\
\text { number of estimators }=300, \\
\text { number of threads }=-1, \\
\text { silent }=\text { True, subsample }=0.9\end{array}$ \\
\hline XGBoost & Model 09 & $\begin{array}{l}\text { learning rate }=0.2, \text { maximum depth }=7 \text {, } \\
\text { number of estimators }=300, \\
\text { number of threads }=-1, \\
\text { silent }=\text { True, subsample }=0.9\end{array}$ \\
\hline XGBoost & Model 10 & $\begin{array}{l}\text { learning rate }=1 \text {, maximum depth }=6 \text {, } \\
\text { number of estimators }=300, \\
\text { number of threads }=-1, \\
\text { silent }=\text { True, subsample }=0.9\end{array}$ \\
\hline Random Forest Classifier & Model 11 & $\begin{array}{l}\text { number of estimators }=300, \\
\text { maximum depth }=300, \text { random state }=27\end{array}$ \\
\hline Random Forest Classifier & Model 12 & $\begin{array}{l}\text { number of estimators }=200, \\
\text { maximum depth }=200, \text { random state }=28\end{array}$ \\
\hline Random Forest Classifier & Model 13 & $\begin{array}{l}\text { number of estimators }=400, \\
\text { maximum depth }=100, \text { random state }=29\end{array}$ \\
\hline
\end{tabular}




\begin{tabular}{|l|l|l|}
\hline Random Forest Classifier & Model 14 & $\begin{array}{l}\text { number of estimators }=300, \\
\text { maximum depth }=300, \\
\text { random state }=30\end{array}$ \\
\hline Random Forest Classifier & Model 15 & $\begin{array}{l}\text { number of estimators }=200, \\
\text { maximum depth }=200, \\
\text { random state }=30\end{array}$ \\
\hline Extra Tree Classifier & Model 16 & $\begin{array}{l}\text { number of estimators }=300, \\
\text { maximum depth }=300, \\
\text { random state }=27\end{array}$ \\
\hline Extra Tree Classifier & Model 17 & $\begin{array}{l}\text { number of estimators }=200, \\
\text { maximum depth }=200, \\
\text { random state }=28\end{array}$ \\
\hline Extra Tree Classifier & Model 18 & $\begin{array}{l}\text { number of estimators }=400, \\
\text { maximum depth }=100, \text { random state }=29\end{array}$ \\
\hline Extra Tree Classifier & Model 19 & $\begin{array}{l}\text { number of estimators }=300, \\
\text { maximum depth }=300, \text { random state }=30\end{array}$ \\
\hline Extra Tree Classifier & Model 20 & $\begin{array}{l}\text { n estimators }=200, \text { maximum depth }=200, \\
\text { random state }=30\end{array}$ \\
\hline Decision Tree Classifier & Model 21 & $\begin{array}{l}\text { maximum depth }=300, \text { criterion }= \\
\text { 'gini' }\end{array}$ \\
\hline Decision Tree Classifier & Model 22 & $\begin{array}{l}\text { maximum depth }=200, \text { criterion }= \\
\text { 'entropy' }\end{array}$ \\
\hline Decision Tree Classifier & Model 23 & $\begin{array}{l}\text { maximum depth }=100, \text { criterion }= \\
\text { gini' }\end{array}$ \\
\hline Decision Tree Classifier & Model 24 & $\begin{array}{l}\text { maximum depth }=90, \text { criterion }= \\
\text { 'gini' }\end{array}$ \\
\hline Decision Tree Classifier & Model 25 & $\begin{array}{l}\text { maximum depth }=90, \text { criterion }= \\
\text { 'entropy' }\end{array}$ \\
\hline
\end{tabular}

Table 3.1: Hybrid Model Parameters

\section{Long-Short Term Memory:}

Long-Short Term Memory (LSTM) is a complex area of deep learning. LSTM is one kind of Recurrent Neural Networks (RNN) that has the ability to learn long-term dependencies, especially in sequence prediction problems. LSTM shows outstanding performance on a large variety of problems. LSTMs are RNNs that differ from traditional feed-forward neural networks and show results that the used machine learning models cannot achieve. LSTMs can avoid the long-term dependency problem. They have a chain like structure where there are repeating interacting modules with four neural networks. The LSTM has cell gate and it can add information to the cell state as well as remove, regulated by structures called gates. Gates in LSTM can let information through.

The gates are composed out of a sigmoid neural network layer and a point wise multiplication operation. The activation function at time $t$ is,

$$
h_{t}=\tanh \left(C_{t}\right) * o_{t}
$$

Output gate modulates the amount of memory content exposure and is expressed 


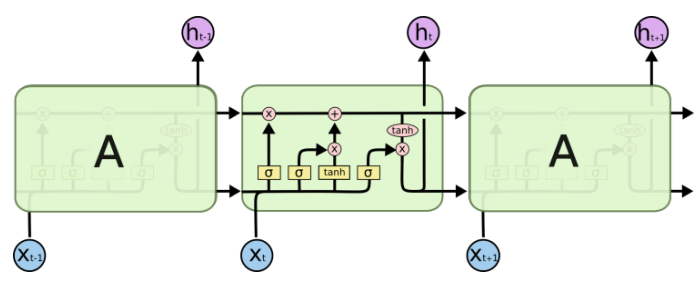

Figure 3.7: LSTM Architecture

as,

$$
o_{t}=\sigma\left(x_{t} U^{o}+h_{t-1} W^{o}\right)
$$

Forget gate which controls the how much of the existing memory to forget and is expressed as,

$$
f_{t}=\sigma\left(x_{t} U^{f}+h_{t-1} W^{f}\right)
$$

Input gate which controls how much of new memory to add is expressed as,

$$
i_{t}=\sigma\left(x_{t} U^{i}+h_{t-1} W^{i}\right)
$$

LSTMs Update existing memory using,

$$
\begin{gathered}
\widetilde{C}_{t}=\sigma\left(x_{t} U^{g}+h_{t-1} W^{g}\right) \text { and } \\
C_{t}=\sigma\left(f_{t} * C_{t-1}+i_{t} * \widetilde{C}_{t}\right)
\end{gathered}
$$

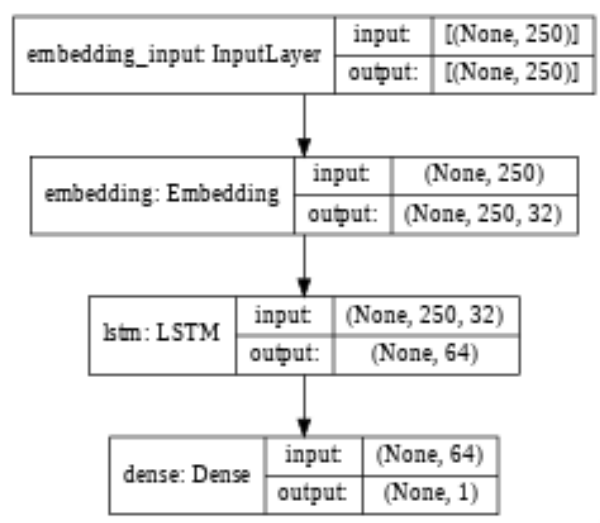

Figure 3.8: Used LSTM Model

We use the Keras library from Tensorflow to apply LSTM. We create a mapping in the form of a counter where we count the occurrence of each word in our corpus to count unique words from the corpus. It helps us define maximum number of words in a specific sequence. We set the maximum length to 20. The reason why we need to define the sequence length is that when we use it with tensorflow, we need to have the same length for each sequence. We create a sequence from tokenizer based on the word indexes. We also pad the sequences to have a specific length (maximum length) for which we use pad_sequence method from Keras. The LSTM architecture requires having sequences of the same length. We use an embedding layer before LSTM architecture and in this way we can get features from the posts contained in 
the datasets. We can map each word to a vector of fixed size with real value element. The idea behind word embedding is that, in contrast to one hot encoding, we use finite size vector to represent a finite size of infinite numbers. The dimensionality of our embedding layer is 32 . Then we compile the model with sigmoid activation function and optimizer is set to Adam. The total number of neurons in the model is 659,201 . While training we performed early stopping to report the results.

\section{Bidirectional Encoder Representations from Transformers (BERT):}

Bidirectional Encoder Representations from Transformers (BERT) is based on Transformersa deep learning model where every output element is connected to every input element and the weightings between them are dynamically calculated based upon their connection. It is an open source machine learning framework for natural language processing tasks. Researchers at Google AI Language open-sourced BERT in 2018 which came as a huge breakthrough and it took the Deep Learning community by storm because of its outstanding performance. BERT is released in two sizes$\mathrm{BERT}_{\mathrm{BASE}}$ which is used to measure the performance of the architecture compara-

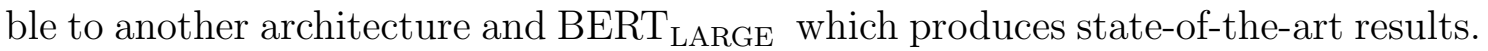
BERT has been pre-trained on- Masked Language Modeling where a percentage of input tokens is masked at random and are predicted and Next Sentence Prediction where given a pair of sentences, it is predicted if the second sentence is the actual next sentence of the first sentence. Below is a BERT model architecture where $\boldsymbol{E}_{1}$ is the embedding representation, $\boldsymbol{T}_{1}$ is the final output and $\boldsymbol{T}_{r}$ are the intermediate representations of the same token. In a 12-layers BERT model a token will have 12 intermediate representations.

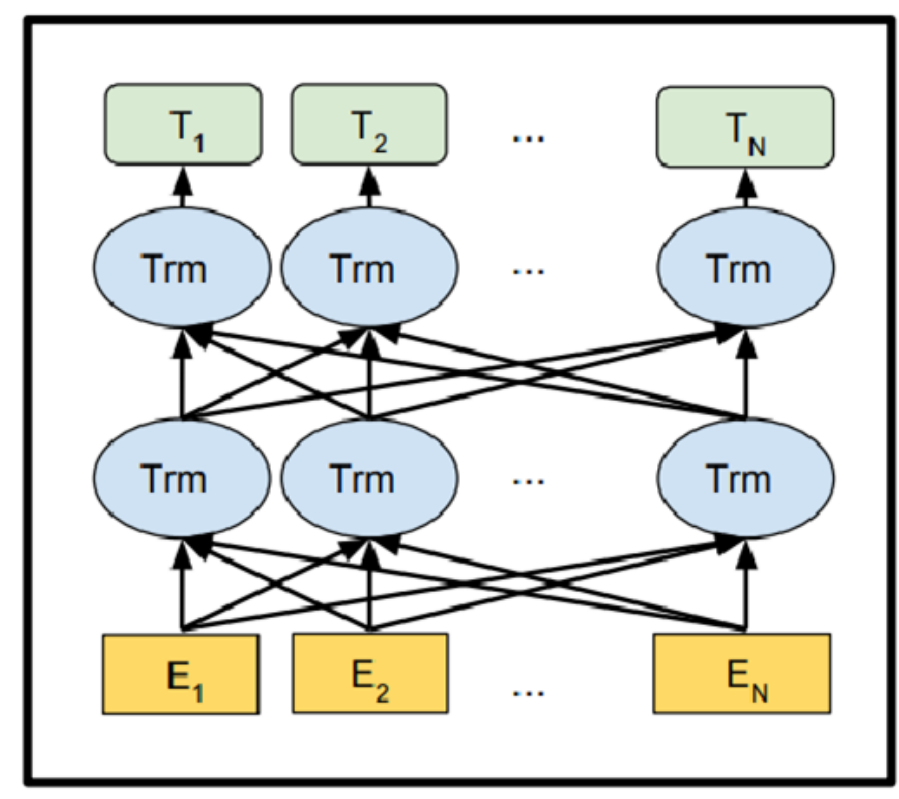

Figure 3.9: BERT Architecture

We use Google's BERT from tensorflow hub. After splitting the datasets into train and test parts, we encode our labels to match the sentiments. The encoder checks for unique classes and transforms that variable into indexes of the classes. The model 
expects three inputs- tokens, input mask and the input type. Before tokenization, we download the BERT model itself showed in the figure below. This is our model represented as only one layer.

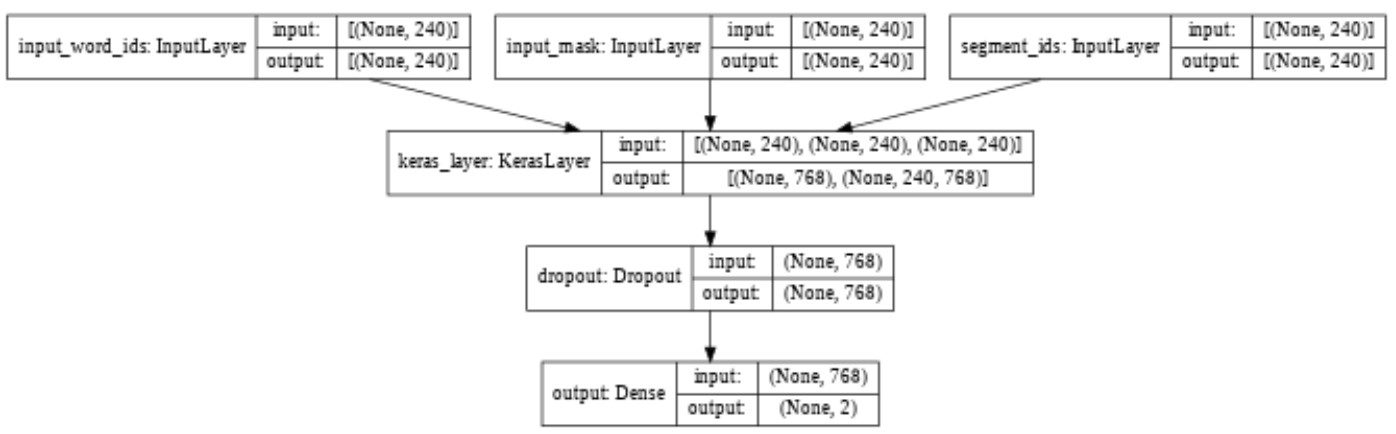

Figure 3.10: Used BERT Model (one layer)

We tokenize the posts and use two additional tokens which are the classification tokens and the separation tokens. The model expects two other inputs which are the input mask and the input type. The input mask allows the model to clearly differentiate between the content and the padding. We need padding to make the posts equal size. We determine the maximum sequence length for this model so that all the inputs are the same length. In the modeling part, along with the three input layers, we use the BERT layer to get two output layers which are the pooled output and the sequence output. We set our output to pooled output only. Then we add a dropout layer which helps us with training and set another dense layer which is the layer for prediction. Also, we set the number of classes and set the activation function as "softmax". The total number of neurons in the model is 177,854,979. For BERT, while training we performed early stopping as well to report the results. 


\subsection{Visualizing Workflow}

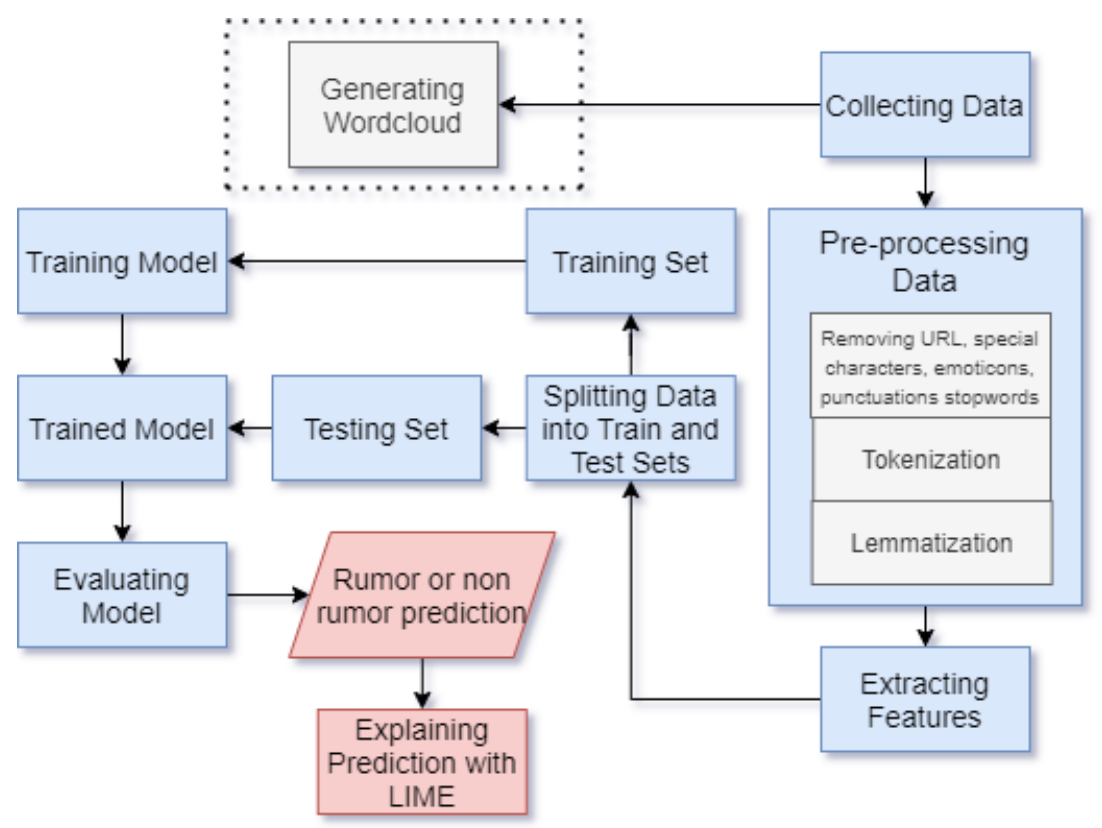

Figure 3.11: Workflow of XGBoost, SVC, RFC, ETC, DTC and Hybrid models

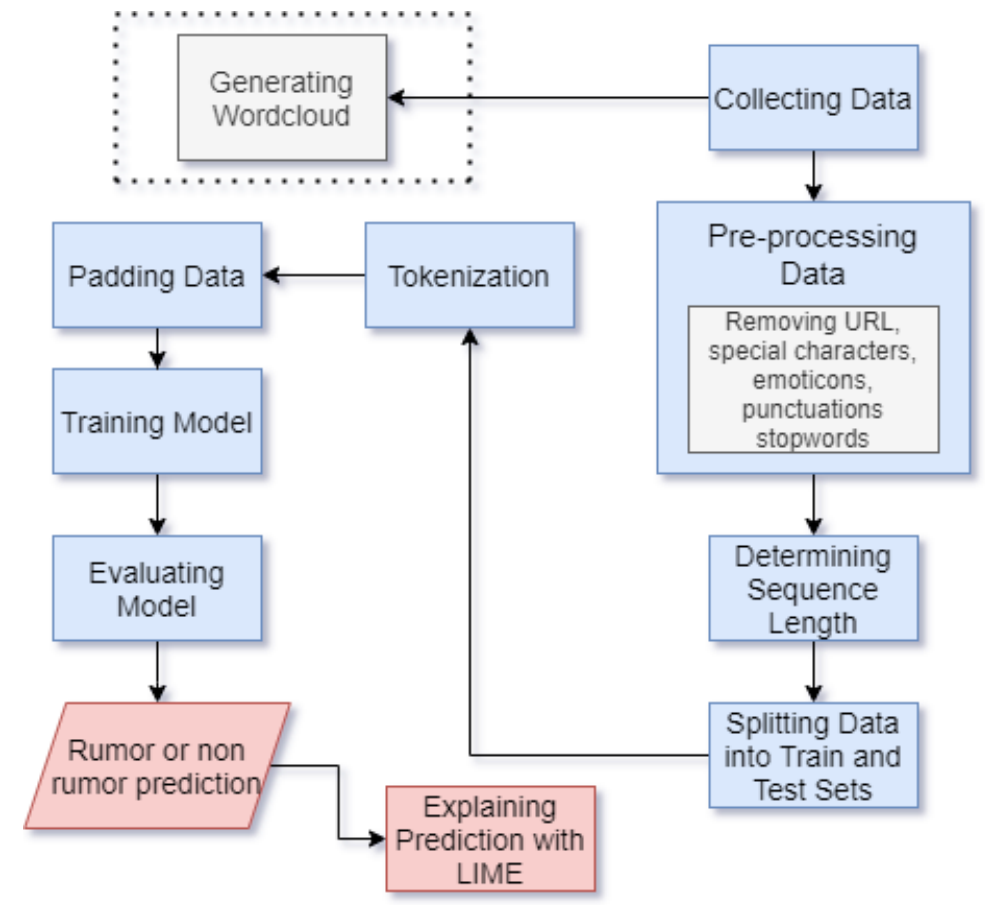

Figure 3.12: Workflow of LSTM and BERT models

The figures aim to provide an insight into how we have done the work for development of our models. 


\section{Chapter 4}

\section{Results and Analysis}

\subsection{Results for Machine Learning Models}

We employed four assessment criteria to assess the performance of the machine learning models: accuracy score, precision score, recall score, and F1 score.

Accuracy: Accuracy is the ratio of correct predictions to total sampled data.

Accuracy $=\frac{\text { Number of } \text { Correct Prediction }}{\text { Total number of predictions made }}$

Accuracy is determined using training data.

F1 Score: The F1 Score represents the Harmonic Mean of precision and recall has a range of $[0,1]$. The precision of the classifier is indicated by it. Higher F1 Score means more accurate our model. It can be stated mathematically as

$$
\mathrm{F}_{1}=2 * \frac{\text { precision } * \text { recall }}{\text { precision }+ \text { recall }}
$$

Precision: Precision denotes the classifiers' precision. It is included within the range $[0,1]$ and is calculated as,

$$
\text { Precision }=\frac{T P}{T P+F P}
$$

where, TP is True Positives, TN is True Negatives, and FP is False Positives.

Recall: Recall provides information about a classifier's completeness. It is included within the range $[0,1]$ and is calculated as,

$$
\text { Recall }=\frac{T P}{T P+F N}
$$


Classification Report of Machine Learning Models on COVID-19 Fake News Dataset:

\begin{tabular}{|l|l|l|l|l|l|}
\hline Model & Accuracy & Label & Precision & Recall & F1 Score \\
\hline XGBoost & \multirow{2}{*}{ S0.23\% } & Rumor & $87.44 \%$ & $93.09 \%$ & $90.06 \%$ \\
\cline { 3 - 6 } & & Non rumor & $93.09 \%$ & $88.00 \%$ & $90.39 \%$ \\
\hline \multirow{2}{*}{ RFC } & \multirow{2}{*}{$90.05 \%$} & Rumor & $87.74 \%$ & $92.00 \%$ & $89.80 \%$ \\
\cline { 3 - 6 } & & Non rumor & $92.34 \%$ & $88.00 \%$ & $90.27 \%$ \\
\hline \multirow{2}{*}{ ETC } & \multirow{2}{*}{ 22.90\% } & Rumor & $88.68 \%$ & $95.00 \%$ & $91.50 \%$ \\
\cline { 3 - 6 } & & Non rumor & $94.68 \%$ & $89.00 \%$ & $91.76 \%$ \\
\hline \multirow{2}{*}{ DTC } & \multirow{2}{*}{ Rumor } & $90.78 \%$ & $95.00 \%$ & $91.50 \%$ \\
\cline { 3 - 6 } & & Non rumor & $94.98 \%$ & $91.00 \%$ & $91.76 \%$ \\
\cline { 3 - 6 } Hybrid & $\mathbf{9 3 . 2 2 \%}$ & Rumor & $84.39 \%$ & $85.00 \%$ & $85.18 \%$ \\
\cline { 3 - 6 } & & Rum rumor & $84.92 \%$ & $84.00 \%$ & $83.44 \%$ \\
\cline { 3 - 6 } & & Non rumor & $\mathbf{9 4 . 2 7 \%}$ & $\mathbf{9 2 . 0 0 \%}$ & $\mathbf{9 3 . 4 7 \%}$ \\
\hline
\end{tabular}

Table 4.1: Accuracy, precision, recall and F1 scores (COVID-19 Fake News Dataset)

Classification Report of Machine Learning Models on Twitter 15 and Twitter 16 Dataset:

\begin{tabular}{|c|c|c|c|c|c|}
\hline Model & Accuracy & Label & Precision & Recall & F1 Score \\
\hline \multirow[t]{2}{*}{ XGBoost } & \multirow[t]{2}{*}{$78.11 \%$} & Rumor & $68.93 \%$ & $83.00 \%$ & $65.70 \%$ \\
\hline & & Non rumor & $82.11 \%$ & $69.00 \%$ & $83.93 \%$ \\
\hline \multirow[t]{2}{*}{ SVM } & \multirow[t]{2}{*}{$81.57 \%$} & Rumor & $84.21 \%$ & $95.00 \%$ & $66.67 \%$ \\
\hline & & Non rumor & $80.83 \%$ & $84.00 \%$ & $87.26 \%$ \\
\hline \multirow[t]{2}{*}{$\mathrm{RFC}$} & \multirow[t]{2}{*}{$75.81 \%$} & Rumor & $93.47 \%$ & $74.00 \%$ & $45.03 \%$ \\
\hline & & Non rumor & $73.71 \%$ & $85.00 \%$ & $84.49 \%$ \\
\hline \multirow[t]{2}{*}{ ETC } & \multirow[t]{2}{*}{$77.88 \%$} & Rumor & $87.69 \%$ & $76.00 \%$ & $92.21 \%$ \\
\hline & & Non rumor & $76.15 \%$ & $91.00 \%$ & $85.41 \%$ \\
\hline \multirow[t]{2}{*}{$\overline{\text { DTC }}$} & \multirow[t]{2}{*}{$76.27 \%$} & Rumor & $69.44 \%$ & $78.00 \%$ & $59.28 \%$ \\
\hline & & Non rumor & $78.52 \%$ & $69.00 \%$ & $83.25 \%$ \\
\hline \multirow[t]{2}{*}{ Hybrid } & \multirow[t]{2}{*}{$82.49 \%$} & Rumor & $87.09 \%$ & $81.00 \%$ & $68.07 \%$ \\
\hline & & Non rumor & $81.23 \%$ & $87.00 \%$ & $87.94 \%$ \\
\hline
\end{tabular}

Table 4.2: Accuracy, precision, recall and F1 scores (concatenation of Twitter 15, Twitter 16 Datasets)

The tables show the results after applying the classification algorithms to each of the test dataset. We can see that the best result is achieved by our hybrid model for the COVID-19 dataset with an accuracy rate of $93.22 \%$ and a weighted average F1 score of $93.01 \%$. And for the Twitter 15 and Twitter 16 datasets, the hybrid model also achieves the best result with an accuracy rate of $82.49 \%$ and a weighted average F1 score of $84 \%$.

To further evaluate the classifiers' performance and decide the results, we have also generated confusion matrices for the algorithms. The general idea of the confusion matrix is to count the number of times instances of one class is classified as instances of another class. In this case, we evaluate how many times the classifiers confused rumors with non-rumors. The horizontal axis is the predicted label and the vertical axis is the true label. 
Confusion matrices of Machine Learning Models on COVID-19 Fake News Dataset:
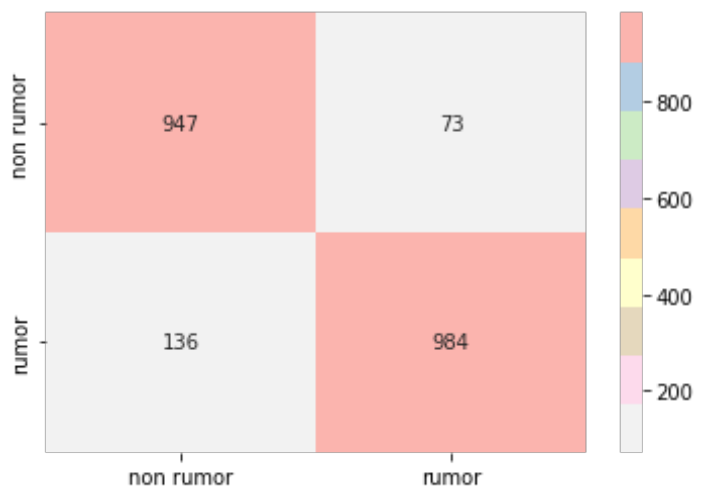

Figure 4.1: Con mat for XGBoost
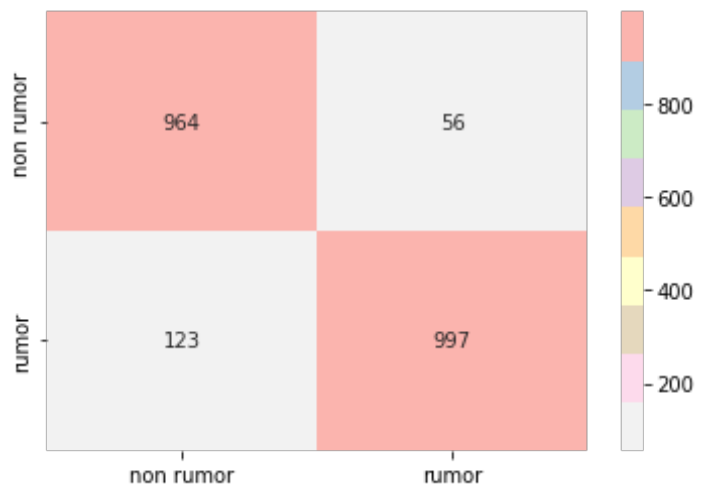

Figure 4.3: Con mat for RFC

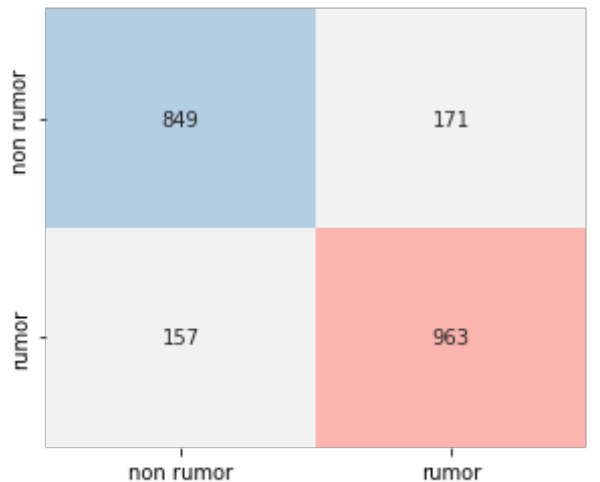

Figure 4.5: Con mat for DTC

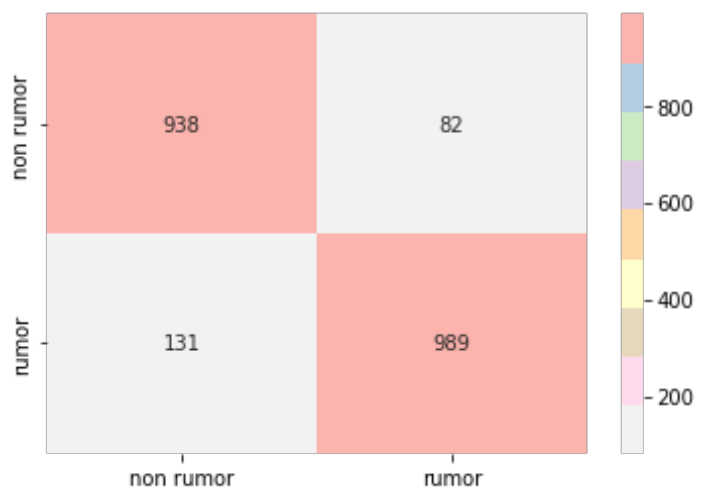

Figure 4.2: Con mat for SVM

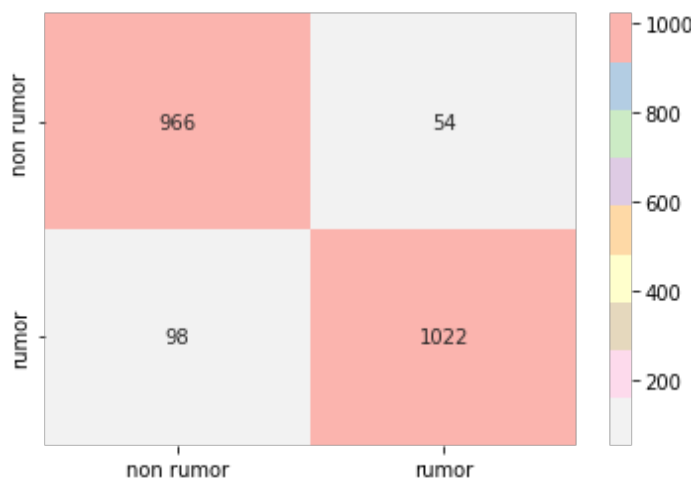

Figure 4.4: Con mat for ETC

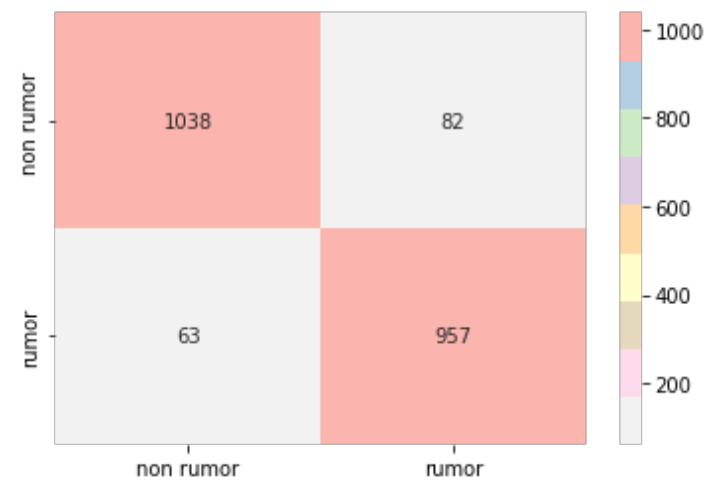

Figure 4.6: Con mat for Hybrid 
From the $25 \%$ of the 8560 posts, XGBoost has been able to successfully detect 984 rumors and 947 non-rumors. 73 posts have been predicted as rumors, although those were non-rumors, and 136 posts have been predicted as non-rumors though those were rumors. SVC has been able to successfully detect 989 rumors and 938 non-rumors. 82 posts have been predicted as rumors, although those were non-rumors, and 131 posts have been predicted as non-rumors though those were rumors. RFC has been able to successfully detect 997 rumors and 964 non-rumors. 56 posts have been predicted as rumors, although those were non-rumors, and 123 posts have been predicted as non-rumors though those were rumors. In ETC has been able to successfully detect 1022 rumors and 966 non-rumors. 54 posts have been predicted as rumors, although those were non-rumors, and 98 posts have been predicted as non-rumors though those were rumors. In DTC has been able to successfully detect 963 rumors and 849 non-rumors. 171 posts have been predicted as rumors, although those were non-rumors, and 157 posts have been predicted as non-rumors though those were rumors and Hybrid Model has been able to successfully detect 957 rumors and 1038 non-rumors. 82 posts have been predicted as rumors, although those were non-rumors, and 63 posts have been predicted as non-rumors though those were rumors.

Confusion matrices of Machine Learning Models on Twitter 15 and Twitter 16 Dataset:

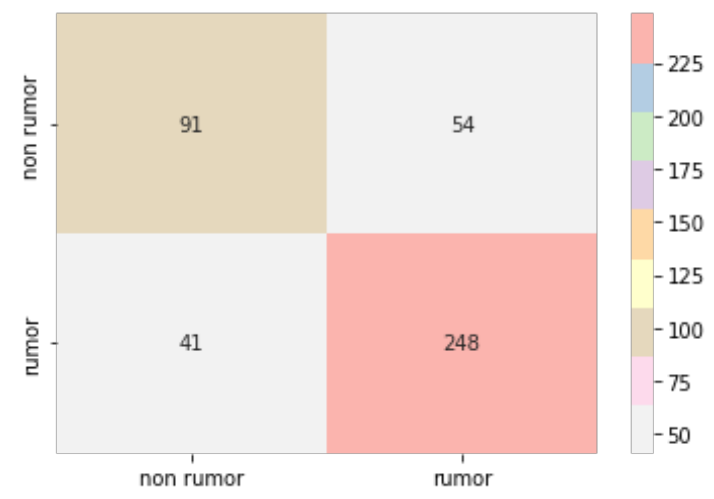

Figure 4.7: Con mat for XGBoost

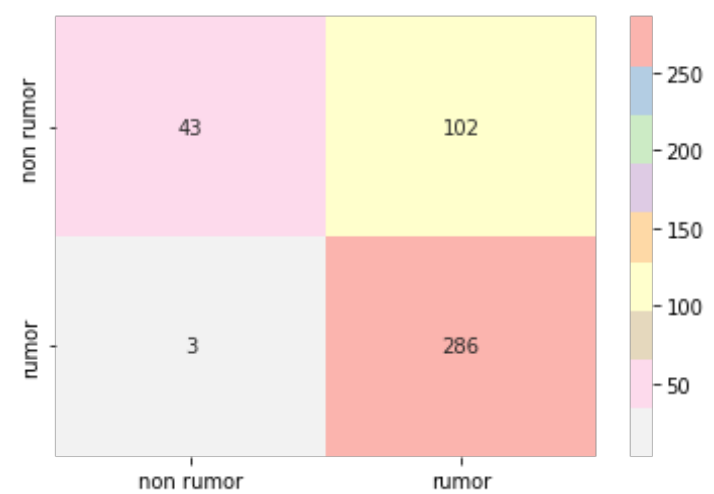

Figure 4.9: Con mat for RFC

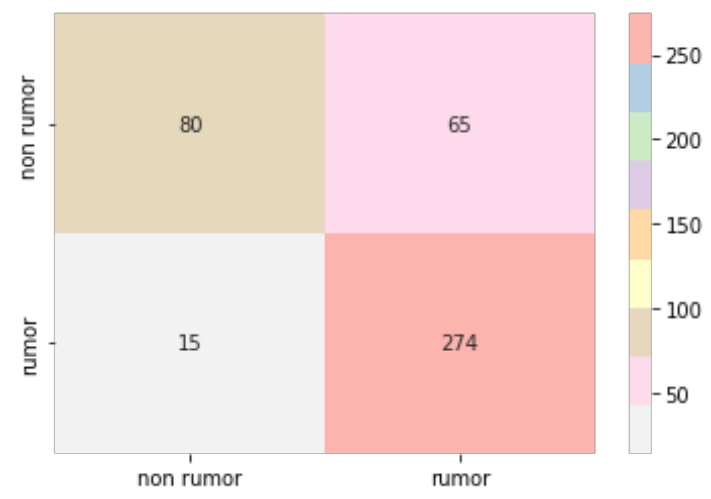

Figure 4.8: Con mat for SVM

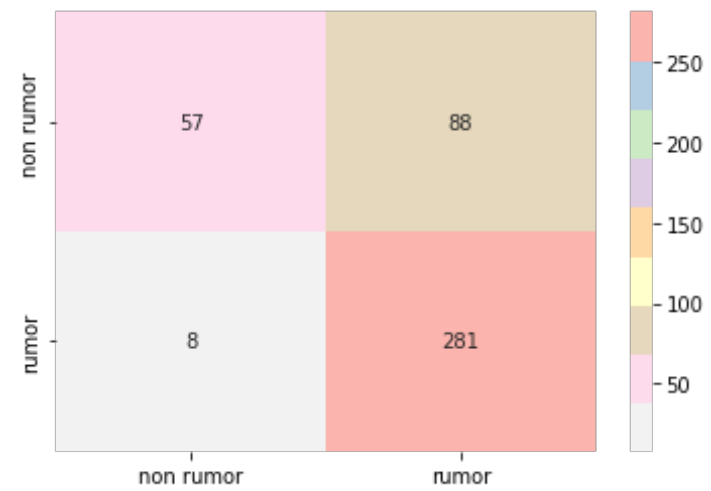

Figure 4.10: Con mat for ETC 


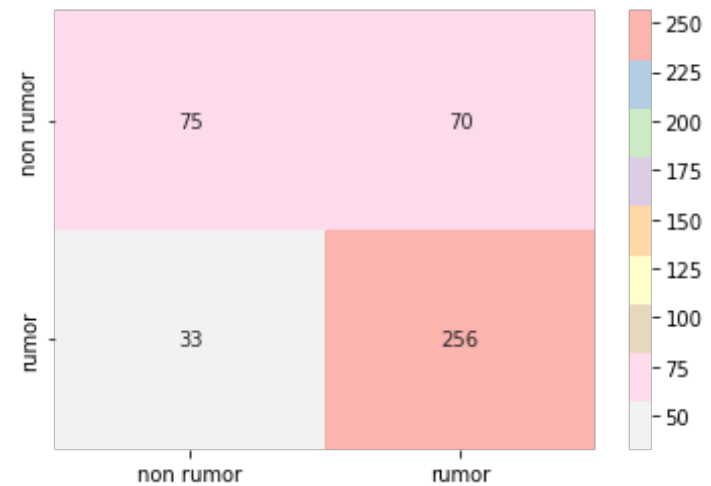

Figure 4.11: Con mat for DTC

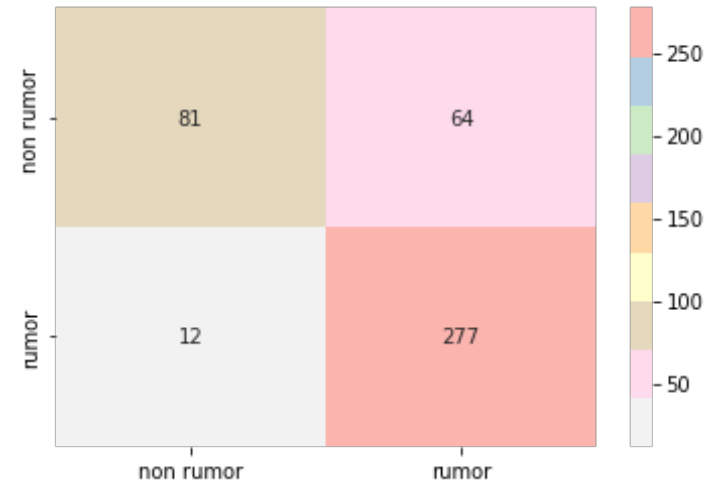

Figure 4.12: Con mat for Hybrid

From the $25 \%$ of the 1736 posts, XGBoost has been able to successfully detect 248 rumors and 91 non-rumors. 54 posts have been predicted as rumors, although those were non-rumors, and 41 posts have been predicted as non-rumors though those were rumors. SVC has been able to successfully detect 274 rumors and 80 non-rumors. 65 posts have been predicted as rumors, although those were nonrumors, and 15 posts have been predicted as non-rumors though those were rumors. RFC has been able to successfully detect 286 rumors and only 43 non-rumors. 102 posts have been predicted as rumors, although those were non-rumors, and only 3 posts have been predicted as non-rumors though those were rumors. In ETC has been able to successfully detect 281 rumors and 57 non-rumors. 88 posts have been predicted as rumors, although those were non-rumors, and 8 posts have been predicted as non-rumors though those were rumors. In DTC has been able to successfully detect 256 rumors and 70 non-rumors. 75 posts have been predicted as rumors, although those were non-rumors, and 33 posts have been predicted as non-rumors though those were rumors and Hybrid Model has been able to successfully detect 277 rumors and 81 non-rumors. 64 posts have been predicted as rumors, although those were non-rumors, and only 12 posts have been predicted as non-rumors though those were rumors.

\subsection{Results for Deep Learning Models}

The assessment criteria for evaluating the performance of LSTM and BERT are their respective accuracy scores, training and validation accuracy and training and validation loss. Interpreting training and validation losses is important to understand the model's performance. A model is well fit if it initially has a higher validation loss which lowers with addition of more training instances. Moreover, as epochs increase, validation loss should decrease and accuracy should increase. If validation loss keeps increasing and accuracy keeps decreasing, it could mean that the model is jamming the values rather than learning. Again, if both validation loss and accuracy keeps increasing, it could mean the model is overfitting. 
For our LSTM model on the COVID-19 dataset, validation loss decreases and validation accuracy increases which indicates that the model is learning properly resulting in an accuracy of $99.81 \%$ and on the Twitter 15 and Twitter 16 dataset after the model gives an accuracy of $98.63 \%$.
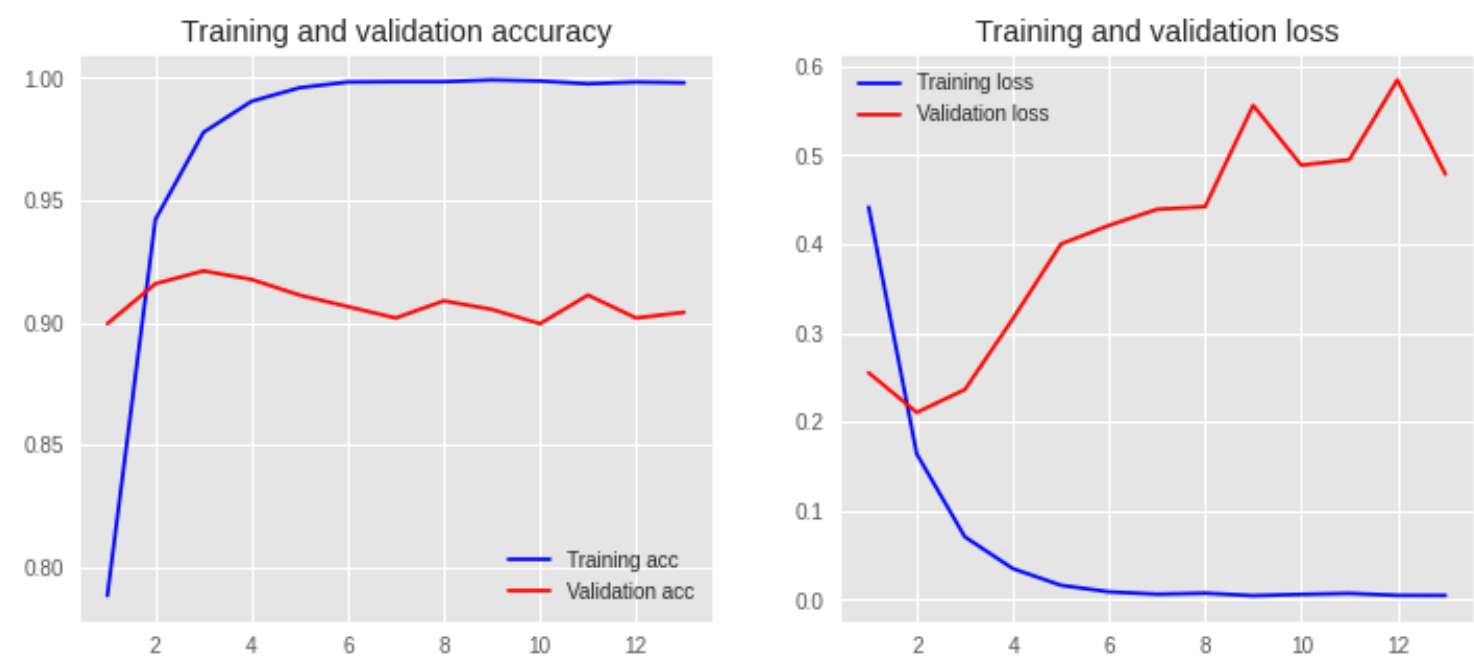

Figure 4.13: Evaluation of LSTM on COVID-19 Dataset
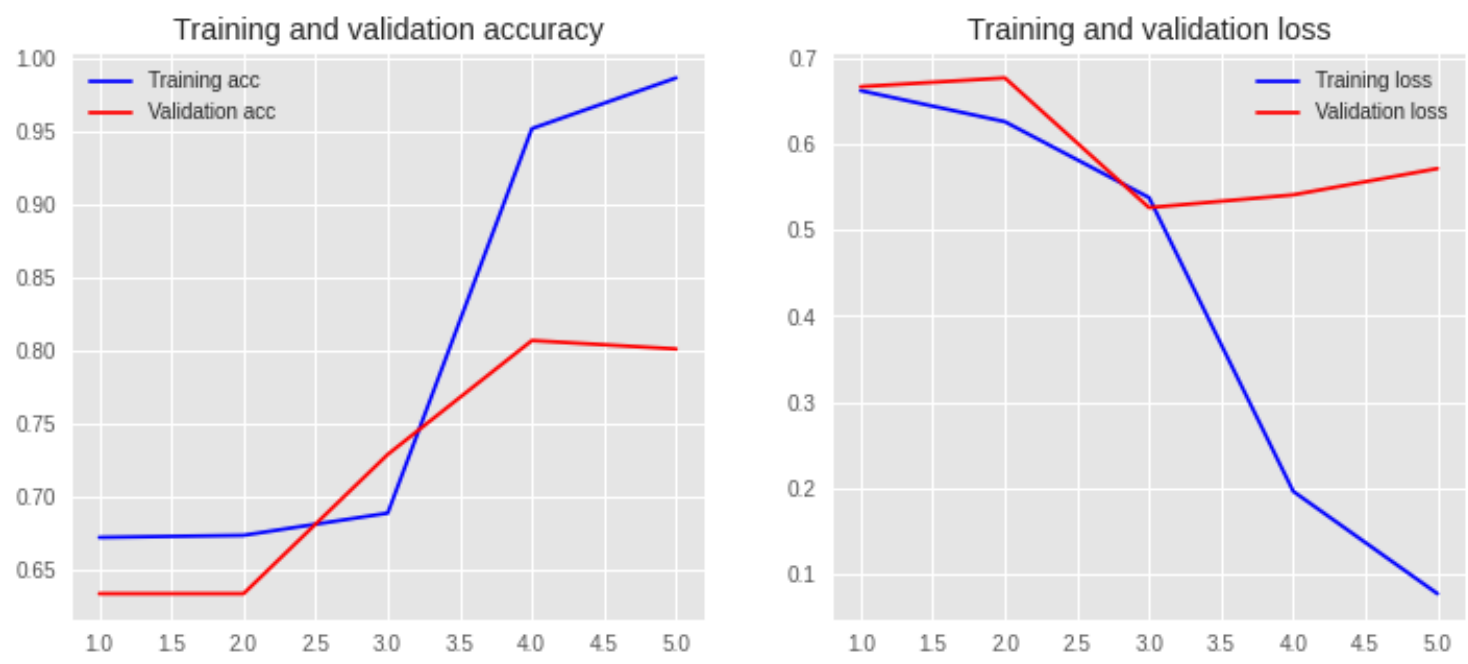

Figure 4.14: Evaluation of LSTM on Twitter 15 and Twitter 16 Dataset

For our BERT model on the COVID-19 and Twitter 15, Twitter 16 datasets, validation loss decreases and validation accuracy increases and the model gives an accuracy of $99.59 \%$ on the COVID-19 dataset and $94.80 \%$ on the Twitter 15 and Twitter 16 datasets. 

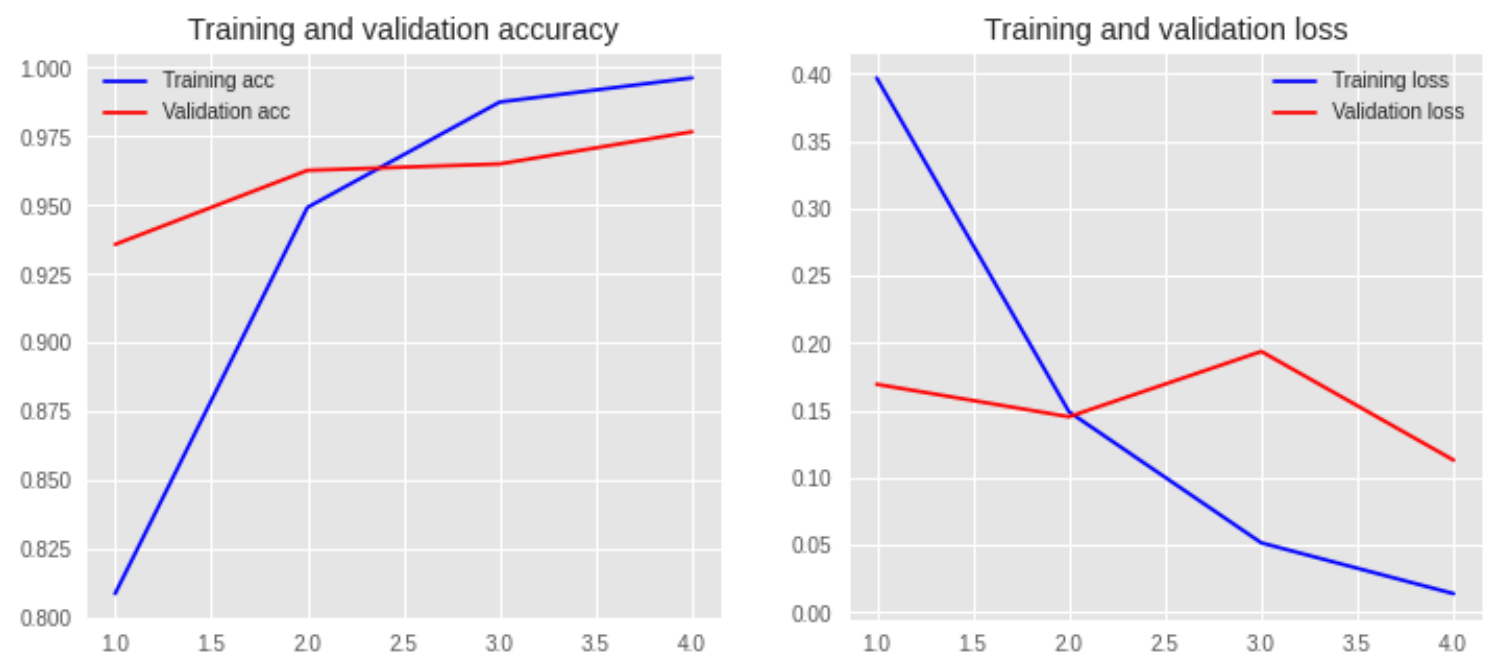

Figure 4.15: Evaluation of BERT on COVID-19 Dataset
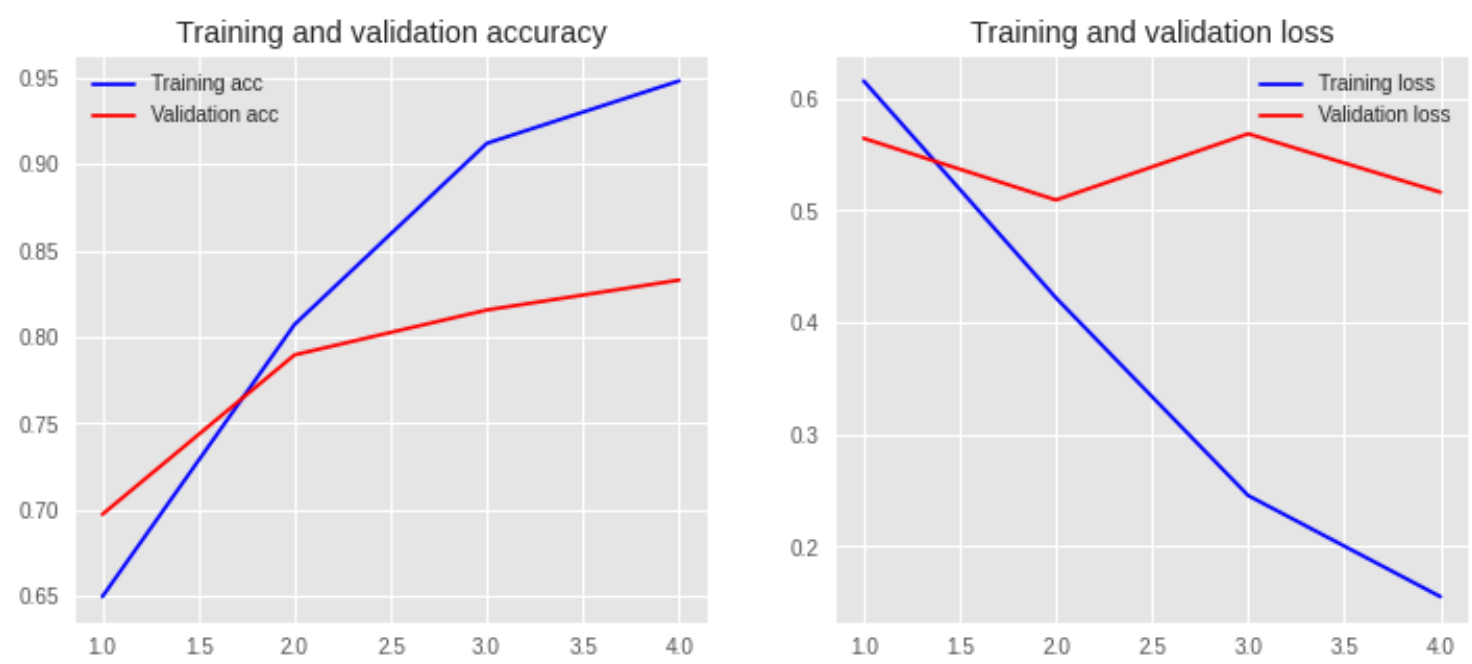

Figure 4.16: Evaluation of BERT on Twitter 15 and Twitter 16 Dataset

The $\mathrm{x}$ axis denotes the number of epochs and the $\mathrm{y}$ axis denotes the percentages. The rates of accuracy are given as tables:

On the COVID-19 Fake News Dataset:

\begin{tabular}{|l|l|}
\hline Model & Accuracy \\
\hline LSTM & $99.81 \%$ \\
\hline BERT & $99.62 \%$ \\
\hline
\end{tabular}

Table 4.3: Accuracy scores (COVID-19 Fake News dataset) 
On the Twitter 15 and Twitter 16 Dataset:

\begin{tabular}{|l|l|}
\hline Model & Accuracy \\
\hline LSTM & $98.41 \%$ \\
\hline BERT & $94.80 \%$ \\
\hline
\end{tabular}

Table 4.4: Accuracy scores (Twitter 15 and Twitter 16 dataset)

\subsection{Decision from Data}

Data with high volume is needed for higher accuracy. Such datasets with relevancy can be quite challenging to manage. In order to provide a fair evaluation for our models, we experimented on two datasets both containing social media posts. As a result, it can be seen that the models gave better accuracy when trained with the COVID-19 Fake News dataset, where the news, opinions, facts are posts (data) from social media than the Twitter 15 and Twitter 16 dataset. In the previous works, these datasets have been used, although the models differ. Our models, specifically the deep learning approach, have provided with benchmark accuracy, though there is still scope to improve the performance of BERT. To our knowledge, the existing works tend more to higher accuracy achievement than explaining the models. But models that are inexplicable can lead to multiple stumbling boxes. Therefore, we felt the need to evaluate the models' decision and visualize with features that contribute to the models' decisions. So, we apply LIME. 


\section{Chapter 5}

\section{Explainable AI}

Models that are not interpretable can raise various problems. According to [37], there are still not a lot of works where the models explain their decision making process and it is not any different for the task of detecting rumors. Therefore, we try to make the predictions more explainable with the help of LIME.

\subsection{Squeezing LIME}

LIME stands for Local Interpretable Model-agnostic Explanations which is an explainable AI method. Its goal is to make the predictions of machine learning models understandable to humans. The method can explain individual instances which makes it suitable for local explanations. LIME manipulates the input data and creates a series of artificial data containing only a part of the original attributes. Thus, in the case of text data, for example, different versions of the original text are created, in which a certain number of different, randomly selected words are removed. This new artificial data is then assigned to different categories (classified). Hence, through the presence or absence of certain keywords we can see their influence on the classification of the selected text. LIME gives the output as list of explanations which reflects the contribution of each feature which resulted in the final prediction. Our datasets have a lot of features. For the purpose of better understanding and simplicity in explanation, we deal with a few top selected features by LIME. We choose a prediction which we want to explain and then LIME creates permutations of that chosen instance and collects the models predictions. Then, weight is assigned to the samples based on how closely they match the data of the original prediction. After that, a new model which is interpretable and less complex is trained on the data variations created using the weights attached to each variation. Thus, the local interpretable model can explain the prediction. We represent the given list of explanations by LIME as graphs for visualization. In the figures, the green bars represent the importance of some of the top features in the models' prediction of a post being rumor and the red bars denote the opposite. The $\mathrm{x}$ axis represents the weights of each feature (positive or negative) and the y axis represents the features. The larger the weight, the greater the importance of the feature in the decision making process of the model. Positive weight means that the machine interprets the post to be a rumor and negative weight means that the machine interprets the post to be a non rumor. 


\subsubsection{LIME on Machine Learning Models}

We consider models with an accuracy of $90 \%$ and above to apply lime. These models are XGBoost, SVC, RFC, ETC and hybrid model trained on the dataset regarding COVID-19.

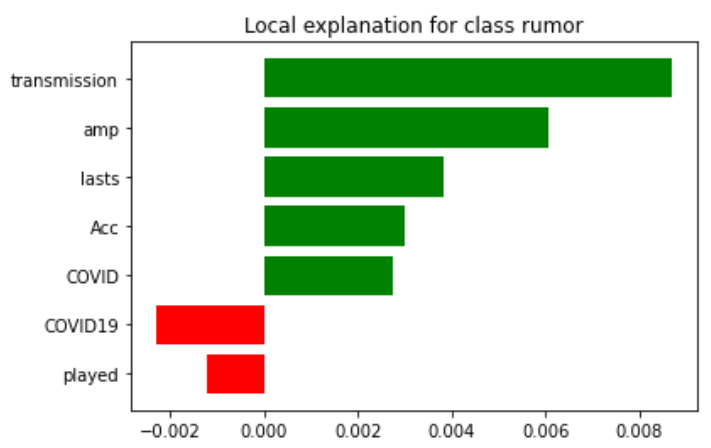

Figure 5.1: XGBoost

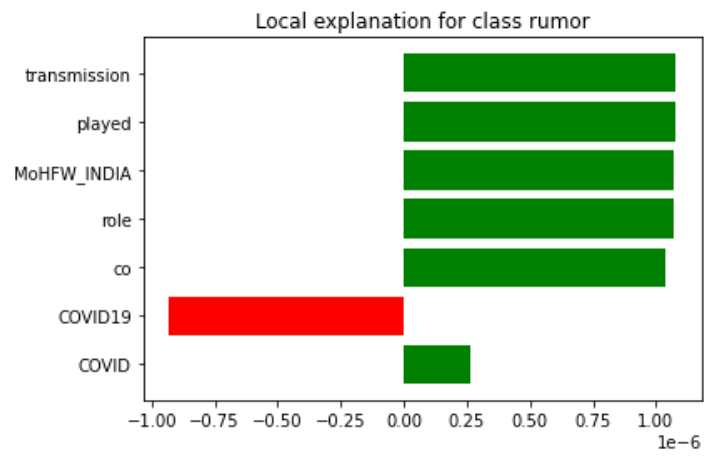

Figure 5.3: RFC

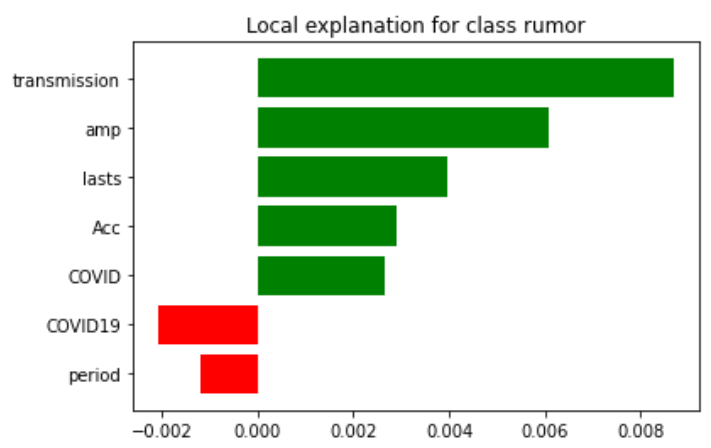

Figure 5.2: SVM

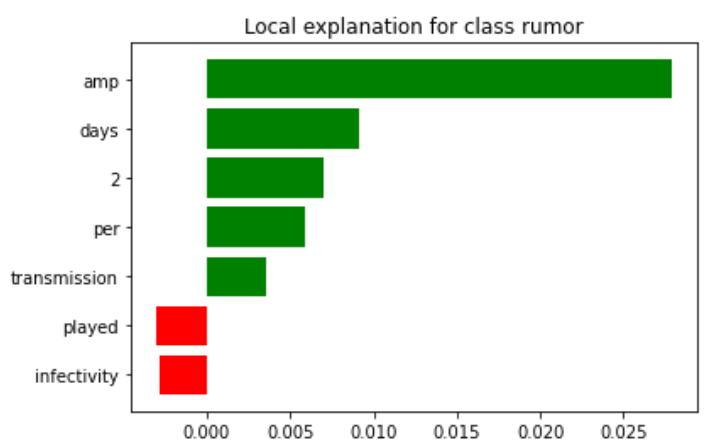

Figure 5.4: ETC

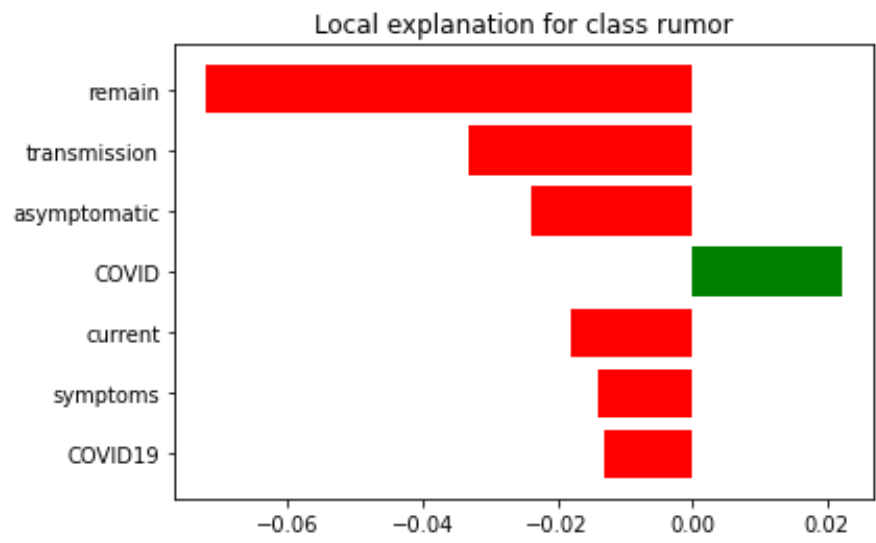

Figure 5.5: Hybrid

For XGBoost, of the seven top features, the feature "transmission" has the most importance in the model's prediction of a post being a rumor and "COVID19" has least importance meaning this feature acts toward the sample to be considered non rumor by the model. For, SVC, it is the same as XGB. RFC considers the top five features almost equally important for a post to be rumor, while "COVID19" has least importance here as well. For, ETC the feature with the most importance is "amp" and least importance is "played". For the hybrid model, the feature 
"COVID" has most importance and and "COVID19" has negative impact. Therefore, from the local explanations, we can see that according to the models, a post is most likely to be a non-rumor and true information if it has the feature "COVID". We then apply lime on XGBoost, SVC, RFC, ETC and hybrid model trained on the public datasets Twitter 15 and Twitter 16. We consider models with accuracy of $77 \%$ and above to apply lime.

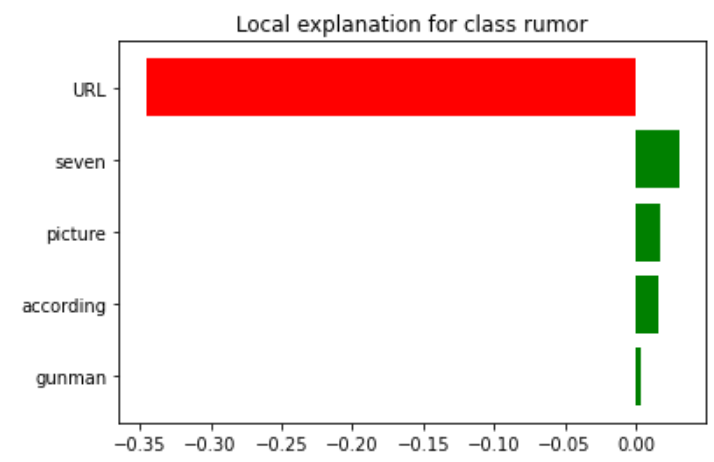

Figure 5.6: XGBoost

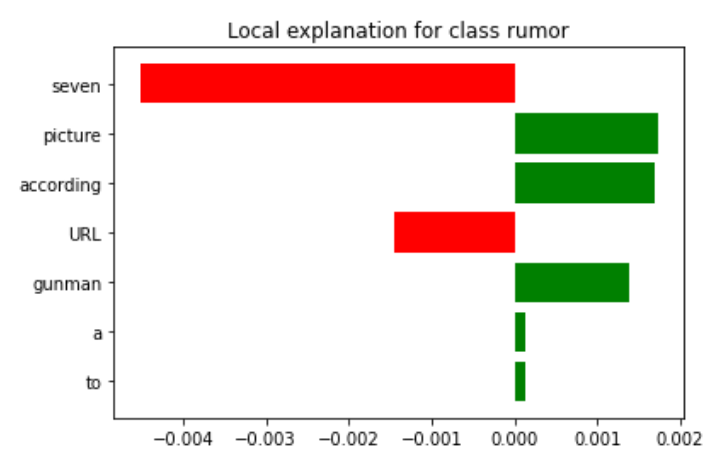

Figure 5.8: ETC

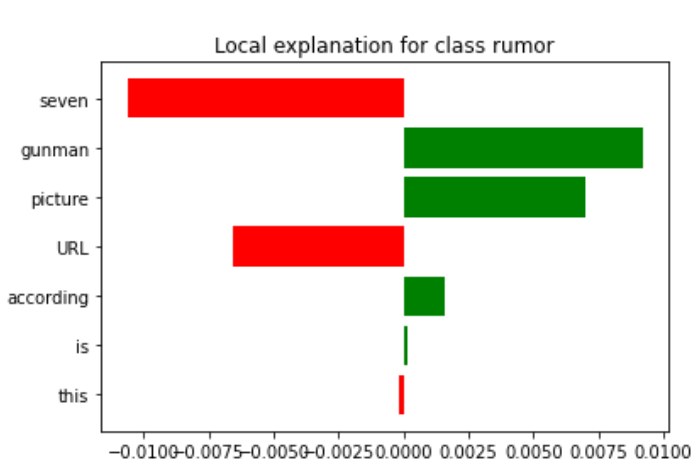

Figure 5.7: SVM

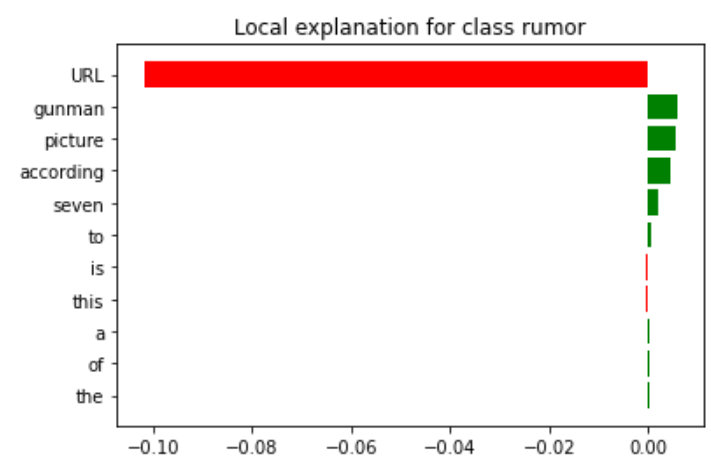

Figure 5.9: Hybrid

For XGBoost, of the seven top features, the feature "seven" has the most importance in the model's prediction of a post being a rumor. For, SVC "gunman" has the most importance in the model's prediction and "URL" has the least importance. ETC considers "picture" and "according" as the most important. For the hybrid model, "gunman", "picture", "according" has almost the same importance while the feature "URL" has a huge negative impact. Therefore, from the local explanations, we can see that according to the machine learning models, a post is most likely to be a non-rumor and may contain true information if it has the feature "URL". This can actually lead to an important observation which is, according to the machine learning models, the COVID-19 related posts containing a URL has a better chance of being a non rumor post.

\subsubsection{LIME on LSTM}

It is challenging to interpret text classifications from word embedding for the lack of LIME documentation. However, our method takes the texts and uses tokenizer 
that we have already fit on the train data. We transform the text into sequences and the sequences are padded using max length. The method returns an array that contains both the predictions for the non rumor class and the rumor class. We apply the method to the explainer and pass a string to the predictor. Below is the result.

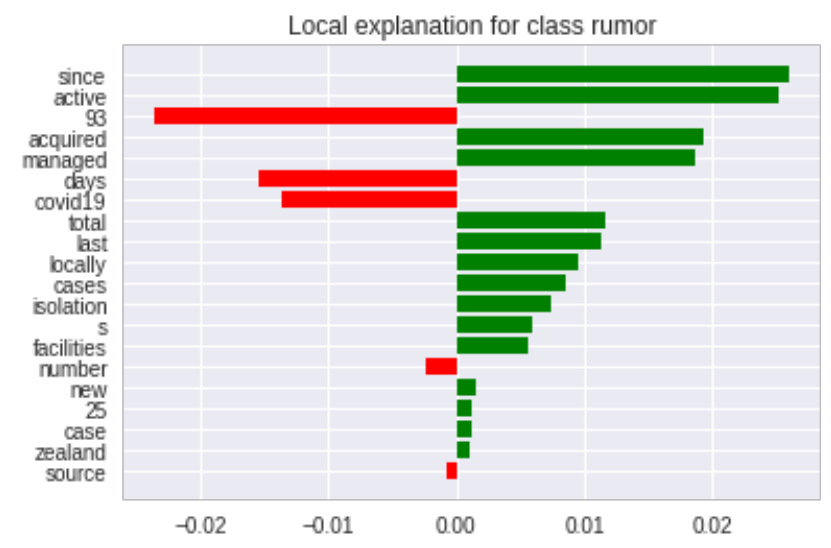

Figure 5.10: LSTM's Explanation (COVID-19 Fake News dataset)

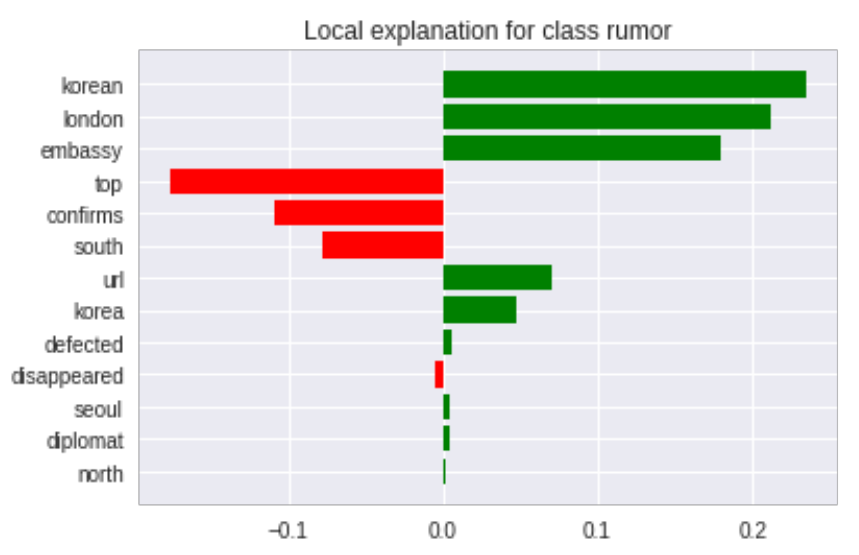

Figure 5.11: LSTM's Explanation (concatenation of Twitter 15 and Twitter 16 dataset)

The result gives us an idea about which features contribute to the consideration of a post being rumor and which features contribute to the consideration of a post being non rumor by LSTM. We choose the last sentence from each dataset to get the explanation. We can see that in the COVID-19 dataset, features like "since", "active", "acquired", "managed" etc. have the most importance in the model's prediction of a post being a rumor and "days", "COVID-19", "number" etc. have least importance meaning this feature acts toward the sample to be considered non rumor by the model.

In the latter dataset, features like "korean", "london", "embassy" etc. have the most importance in the model's prediction of a post being a rumor and "top", "confirms", "south" etc. have least importance meaning this feature acts toward the sample to be considered non rumor by the model. 
Models with higher accuracy illustrate which feature contributes the most for a post from social to be predicted as a rumor or a non rumor thus demystifying the black box learning models. 


\section{Chapter 6}

\section{Future Work Plan and Conclusion}

\subsection{Scopes in the Future}

Our future work concerns a deeper analysis of the datasets and adding new research components that are propaganda detection and multi-lingual rumor detection. So far, we have predicted Rumor and non-rumor with a satisfactory accuracy rate using multiple models and our hybrid models, but in the future,

i) Better predictive performance can be achieved than that which could be obtained using the hybrid model on the Twitter 15 and Twitter 16 datasets.

ii) The performance of BERT can be improved.

iii) Spreading of rumor can be reduced if the earliest form of rumor which is propaganda can be detected.

iv) A mobile app for rumor detection can be developed to prevent rumors and hateful information from spreading which in turn can prevent cyber bullying.

\subsection{Conclusion}

To conclude, as the alteration of information is one of the oldest problems in human civilization, it has become tough to detect rumors in this digital era. As a result, the consequences of these rumors are becoming devastating as they can fuel rage among mass people. The whole life circle of rumors can be stopped by detecting and removing rumors early. In this paper, we tried to find an efficient way to detect rumors and non-rumors. We collected labeled datasets each containing thousands of social media posts and predicted the results using machine learning and deep learning algorithms along with a hybrid model, and compared the results. Among the algorithms, our hybrid model on COVID-19 dataset and LSTM on both the datasets showed superior performance and we are planning to utilize this information in the future by using different modeling algorithms to detect rumors, non-rumors, and also the earliest form of rumor which is propaganda. Finally, we put emphasis on the need to use explainable AI so that human-reliable knowledge can be extracted. 


\section{Bibliography}

[1] T. Chen, X. Li, H. Yin, and J. Zhang, "Call attention to rumors: Deep attention based recurrent neural networks for early rumor detection," in PacificAsia conference on knowledge discovery and data mining, Springer, 2018, pp. 4052.

[2] N. Grinberg, K. Joseph, L. Friedland, B. Swire-Thompson, and D. Lazer, "Fake news on twitter during the 2016 us presidential election," Science, vol. 363, no. 6425, pp. 374-378, 2019.

[3] S. Vosoughi, D. Roy, and S. Aral, "The spread of true and false news online," Science, vol. 359, no. 6380, pp. 1146-1151, 2018.

[4] S. Kwon, M. Cha, K. Jung, W. Chen, and Y. Wang, "Prominent features of rumor propagation in online social media," in 2013 IEEE 13th international conference on data mining, IEEE, 2013, pp. 1103-1108.

[5] P. by Statista Research Department and S. 10, Social network penetration worldwide from 2017 to 2025, Sep. 2021. [Online]. Available: https://www. statista.com/statistics/278414/number-of-worldwide-social-network-users/.

[6] S. Kwon, M. Cha, and K. Jung, "Rumor detection over varying time windows," PloS one, vol. 12, no. 1, e0168344, 2017.

[7] L. Li, G. Cai, and N. Chen, "A rumor events detection method based on deep bidirectional gru neural network," in 2018 IEEE 3rd International Conference on Image, Vision and Computing (ICIVC), IEEE, 2018, pp. 755-759.

[8] J. Cao, J. Guo, X. Li, Z. Jin, H. Guo, and J. Li, "Automatic rumor detection on microblogs: A survey," arXiv preprint arXiv:1807.03505, 2018.

[9] C. Castillo, M. Mendoza, and B. Poblete, "Information credibility on twitter," in Proceedings of the 20th international conference on World wide web, 2011, pp. 675-684.

[10] Z. Jin, J. Cao, Y. Zhang, and Y. Zhang, "Mcg-ict at mediaeval 2015: Verifying multimedia use with a two-level classification model.," in MediaEval, 2015.

[11] Z. Jin, J. Cao, Y. Zhang, and J. Luo, "News verification by exploiting conflicting social viewpoints in microblogs," in Proceedings of the AAAI Conference on Artificial Intelligence, vol. 30, 2016.

[12] T. G. Dietterich, "Ensemble methods in machine learning," in International workshop on multiple classifier systems, Springer, 2000, pp. 1-15.

[13] E. Bauer and R. Kohavi, "An empirical comparison of voting classification algorithms: Bagging, boosting, and variants," Machine learning, vol. 36, no. 1, pp. 105-139, 1999. 
[14] L. Xu, A. Krzyzak, and C. Y. Suen, "Methods of combining multiple classifiers and their applications to handwriting recognition," IEEE transactions on systems, man, and cybernetics, vol. 22, no. 3, pp. 418-435, 1992.

[15] S. Liu, Y. Wang, J. Zhang, C. Chen, and Y. Xiang, "Addressing the class imbalance problem in twitter spam detection using ensemble learning," Computers 65 Security, vol. 69, pp. 35-49, 2017.

[16] M. Al-Sarem, W. Boulila, M. Al-Harby, J. Qadir, and A. Alsaeedi, "Deep learning-based rumor detection on microblogging platforms: A systematic review," IEEE Access, vol. 7, pp. 152 788-152 812, 2019.

[17] S. Yang, J. Jiang, A. Pal, K. Yu, F. Chen, and S. Yu, "Analysis and insights for myths circulating on twitter during the covid-19 pandemic," IEEE Open Journal of the Computer Society, vol. 1, pp. 209-219, 2020.

[18] G. Shrivastava, P. Kumar, R. P. Ojha, P. K. Srivastava, S. Mohan, and G. Srivastava, "Defensive modeling of fake news through online social networks," IEEE Transactions on Computational Social Systems, vol. 7, no. 5, pp. 11591167, 2020.

[19] T. Saha, S. R. Jayashree, S. Saha, and P. Bhattacharyya, "Bert-caps: A transformer-based capsule network for tweet act classification," IEEE Transactions on Computational Social Systems, vol. 7, no. 5, pp. 1168-1179, 2020.

[20] D. Shah and T. Zaman, "Rumors in a network: Who's the culprit?" IEEE Transactions on information theory, vol. 57, no. 8, pp. 5163-5181, 2011.

[21] K. Zhu and L. Ying, "Information source detection in the sir model: A samplepath-based approach," IEEE/ACM Transactions on Networking, vol. 24, no. 1, pp. 408-421, 2014.

[22] J. Jiang, S. Wen, S. Yu, Y. Xiang, and W. Zhou, "K-center: An approach on the multi-source identification of information diffusion," IEEE Transactions on Information Forensics and Security, vol. 10, no. 12, pp. 2616-2626, 2015.

[23] B. Malhotra and D. K. Vishwakarma, "Classification of propagation path and tweets for rumor detection using graphical convolutional networks and transformer based encodings," in 2020 IEEE Sixth International Conference on Multimedia Big Data (BigMM), IEEE, 2020, pp. 183-190.

[24] T. Chen, H. Chen, and X. Li, "Rumor detection via recurrent neural networks: A case study on adaptivity with varied data compositions," in PacificAsia Conference on Knowledge Discovery and Data Mining, Springer, 2018, pp. $121-127$.

[25] A. E. Fard, M. Mohammadi, Y. Chen, and B. Van de Walle, "Computational rumor detection without non-rumor: A one-class classification approach," IEEE Transactions on Computational Social Systems, vol. 6, no. 5, pp. 830-846, 2019.

[26] H. Guo, J. Cao, Y. Zhang, J. Guo, and J. Li, "Rumor detection with hierarchical social attention network," in Proceedings of the 27th ACM International Conference on Information and Knowledge Management, 2018, pp. 943-951. 
[27] S. Singhal, R. R. Shah, T. Chakraborty, P. Kumaraguru, and S. Satoh, "Spotfake: A multi-modal framework for fake news detection," in 2019 IEEE fifth international conference on multimedia big data (BigMM), IEEE, 2019, pp. 3947.

[28] Z. Jin, J. Cao, H. Guo, Y. Zhang, and J. Luo, "Multimodal fusion with recurrent neural networks for rumor detection on microblogs," in Proceedings of the 25th ACM international conference on Multimedia, 2017, pp. 795-816.

[29] J. P. Singh, A. Kumar, N. P. Rana, and Y. K. Dwivedi, "Attention-based lstm network for rumor veracity estimation of tweets," Information Systems Frontiers, pp. 1-16, 2020.

[30] F. M. Dito, H. A. Alqadhi, and A. Alasaadi, "Detecting medical rumors on twitter using machine learning," in 2020 International Conference on Innovation and Intelligence for Informatics, Computing and Technologies (3ICT), IEEE, 2020, pp. 1-7.

[31] Q. Li, Q. Zhang, and L. Si, "Rumor detection by exploiting user credibility information, attention and multi-task learning," in Proceedings of the 57th Annual Meeting of the Association for Computational Linguistics, 2019, pp. 11731179.

[32] Y. Chen, J. Sui, L. Hu, and W. Gong, "Attention-residual network with cnn for rumor detection," in Proceedings of the 28th ACM International Conference on Information and Knowledge Management, 2019, pp. 1121-1130.

[33] W. Chen, Y. Zhang, C. K. Yeo, C. T. Lau, and B. S. Lee, "Unsupervised rumor detection based on users' behaviors using neural networks," Pattern Recognition Letters, vol. 105, pp. 226-233, 2018.

[34] T. Chen, X. Li, H. Yin, and J. Zhang, "Call attention to rumors: Deep attention based recurrent neural networks for early rumor detection," in PacificAsia conference on knowledge discovery and data mining, Springer, 2018, pp. 4052.

[35] J. Ma, W. Gao, and K.-F. Wong, "Detect rumors in microblog posts using propagation structure via kernel learning," Association for Computational Linguistics, 2017.

[36] S. Zamani, M. Asadpour, and D. Moazzami, "Rumor detection for persian tweets," in 2017 Iranian Conference on Electrical Engineering (ICEE), IEEE, 2017, pp. 1532-1536.

[37] C. Zucco, H. Liang, G. Di Fatta, and M. Cannataro, "Explainable sentiment analysis with applications in medicine," in 2018 IEEE International Conference on Bioinformatics and Biomedicine (BIBM), IEEE, 2018, pp. 1740-1747. 